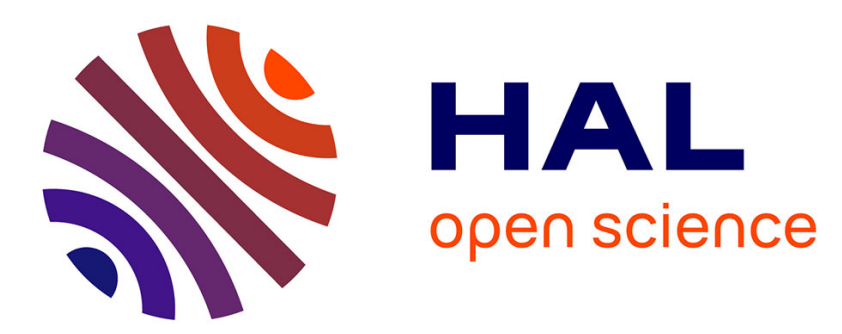

\title{
A FETI method with a mesh independent condition number for the iteration matrix
}

Christine Bernardi, Tomas Chacon-Rebollo, Eliseo Chacòn Vera

\section{To cite this version:}

Christine Bernardi, Tomas Chacon-Rebollo, Eliseo Chacòn Vera. A FETI method with a mesh independent condition number for the iteration matrix. Computer Methods in Applied Mechanics and Engineering, 2008, 197 (issues-13-16), pp.1410-1429. 10.1016/j.cma.2007.11.019 . hal-00170169

\section{HAL Id: hal-00170169 \\ https://hal.science/hal-00170169}

Submitted on 6 Sep 2007

HAL is a multi-disciplinary open access archive for the deposit and dissemination of scientific research documents, whether they are published or not. The documents may come from teaching and research institutions in France or abroad, or from public or private research centers.
L'archive ouverte pluridisciplinaire HAL, est destinée au dépôt et à la diffusion de documents scientifiques de niveau recherche, publiés ou non, émanant des établissements d'enseignement et de recherche français ou étrangers, des laboratoires publics ou privés. 


\title{
A FETI method with a mesh independent condition number for the iteration matrix
}

\author{
C. Bernardi ${ }^{1}, \mathrm{~T}$. Chacón Rebollo², E. Chacón Vera ${ }^{2}$
}

August 21, 2007

\begin{abstract}
We introduce a framework for FETI methods using ideas from the decomposition via Lagrange multipliers of $H_{0}^{1}(\Omega)$ derived by Raviart-Thomas [22] and complemented with the detailed work on polygonal domains developed by Grisvard [17]. We compute the action of the Lagrange multipliers using the natural $H_{00}^{1 / 2}$ scalar product, therefore no consistency error appears. As a byproduct, we obtain that the condition number for the iteration matrix is independent of the mesh size and there is no need for preconditioning. This result improves the standard asymptotic bound for this condition number shown by Mandel-Tezaur in [19]. Numerical results that confirm our theoretical analysis are presented.
\end{abstract}

Résumé. Nous proposons une nouvelle approche des méthodes FETI: la décomposition de domaine fait appel aux multiplicateurs de Lagrange tels qu'introduits par Raviart-Thomas [22] et au traitement des domaines polygonaux dû à Grisvard [17]. Ces multiplicateurs utilisent le produit scalaire de $H_{00}^{1 / 2}$, de sorte qu'aucune erreur de consistance n'apparaît. En outre, nous prouvons que le nombre de condition de la matrice liée à chaque itération est indépendant de la taille du maillage, ce qui améliore le résultat de MandelTezaur [19]; par suite, aucun préconditionnement n'est nécessaire. Nous présentons des expériences numériques qui confirment notre analyse.

\footnotetext{
${ }^{1}$ Laboratoire Jacques-Louis Lions, C.N.R.S. et université Pierre et Marie Curie, b.c. 187, 4 place Jussieu 75252 Paris Cedex 05, France, email: bernardi@ann.jussieu.fr

${ }^{2}$ Dpto. Ecuaciones Diferenciales y Análisis Numérico, Facultad de Matemáticas, Universidad de Sevilla, Tarfia sn. 41012 Sevilla, Spain, email: chacon@us.es, eliseo@us.es Corresponding author: E. Chacón Vera, eliseo@us.es
} 



\section{Introduction}

The Lagrange multiplier formulation for elliptic Dirichlet boundary value problems is a classical technique to handle many difficulties such as highorder equations, the divergence-free constraint or non standard boundary conditions. We are interested here in its applications to domain decomposition methods, more precisely to the Finite Element Tearing and Interconnecting (FETI) method hinted by Dihn, Glowinsky and Periaux [16] in 1983, Dorr [9] in 1988, Roux [24]-[25] in 1989 and further developed by Farhat-Roux and collaborators [10]-[12]-[13]-[14]-[18]-[19]-[20]. This method has been implemented for large scale engineering problems with excellent results, see for instance [8]-[11]-[23].

The idea of the FETI method is to decompose the computational domain into non-overlapping subdomains and to enforce continuity on subdomain interfaces by Lagrange multipliers. Eliminating the subdomain variables yields a dual problem for the Lagrange multipliers, which can be solved by any iteration method. When the preconditioned conjugate gradient method is used we encounter the standard FETI method.

Efficient computations of the Lagrange multipliers is of great interest and has been considered from several points of view. For instance, BarbosaHughes [3] used mesh dependent scalar products, Ben Belgacem [4] used some projection operator onto the $L^{2}$ space on the interfaces and Bertoluzza [5] used wavelet bases.

In this work we introduce a framework for FETI methods using ideas from the decomposition via Lagrange multipliers of $H_{0}^{1}(\Omega)$ derived by RaviartThomas [22] and complemented with the detailed work on polygonal domains developed by Grisvard [17]. As a consequence, we obtain a characterization of $H_{0}^{1}(\Omega)$ more precise than the one in [22]. Our main ingredient next is

the direct computation of the duality $H_{00}^{-1 / 2}-H_{00}^{1 / 2}$ using the natural $H_{00}^{1 / 2}$ scalar product; therefore no consistency error appears. Our analysis allows to deal with cross points and floating subdomains in a natural manner: cross points are dealt with implicitly and the ellipticity on floating subdomains holds naturally because we restrict our work to a subspace that contains the solution and where this ellipticity is satisfied. As a byproduct, we obtain that the condition number for the iteration matrix is independent of the mesh size and does not need any preconditioning. This result improves the standard asymptotic bound for this condition number given by $(1+\log (H / h))^{2}$, where $H$ and $h$ are the characteristic subdomain size and element size respectively, 
shown by Mandel-Tezaur in [19].

The way to approximate the nullity of the jump is of course the main feature of domain decomposition methods. In most cases, the jump is enforced either to cancel in a finite number of nodes (pointwise matching) or to be orthogonal to a finite-dimensional space for the scalar product of $L^{2}(\Gamma)$ (integral matching), see for instance Bernardi-Maday-Patera [6] and the references therein for a large number of applications. The approach that we follow here is rather different and relies on the fact that the jump through $\Gamma$ belongs to the space $H_{00}^{1 / 2}(\Gamma)$. So, this jump is enforced to be orthogonal to an appropriate subspace of $H_{00}^{1 / 2}(\Gamma)$ for the scalar product of $H_{00}^{1 / 2}(\Gamma)$. The main advantages are that:

1. This condition can be written in the continuous case in a natural way: If $(\cdot, \cdot)_{1 / 2,00, \Gamma}$ denotes the scalar product of $H_{00}^{1 / 2}(\Gamma)$ it reads as

$$
\forall \mu \in H_{00}^{1 / 2}(\Gamma), \quad\left([v]_{\Gamma}, \mu\right)_{1 / 2,00, \Gamma}=0
$$

where $[v]_{\Gamma}$ is the jump of $v$ across $\Gamma$.

2. Introducing a Lagrange multiplier to handle this condition is natural. Moreover, due to the intrinsic ellipticity of the scalar products $(\cdot, \cdot)_{1 / 2,00, \Gamma}$ on $H_{00}^{1 / 2}(\Gamma)$, the corresponding mixed problem is well-posed.

3. Then, a discrete problem can easily be constructed by the Galerkin method, so that no consistency error appears from the discretization.

On the other hand, the application of the classical Uzawa's Method or the Conjugate Gradient Method to the dual problem that computes the correct Lagrangian multipliers yield non overlapping domain decomposition methods. The convergence properties of these methods are the same as the ones of the iterative method considered: geometrically convergent in both cases and with a better ratio in the case of Conjugate Gradient Method. Moreover, when performing a finite element approximation of each subproblem, these convergence properties are preserved with a mesh independent ratio thanks to the finite element extension theorems, see for instance BernardiMaday-Rapetti [7]. To avoid more technical details, for the moment we fix our attention on dimension two but we believe the extension to three dimensional problems is just a technical matter.

This work is structured as follows: In Section 2 we present a characterization of $H_{0}^{1}(\Omega)$ via Lagrange multipliers and in terms of the $H_{00}^{1 / 2}$ jumps across 
interfaces. Then we remind the primal hybrid formulation or Lagrangian formulation for elliptic problems with Dirichlet boundary conditions. This formulation requires the use of the duality $H_{00}^{-1 / 2}-H_{00}^{1 / 2}$ on each interface; in Section 3 we reformulate the method via Riesz representation so that this duality is replaced by the scalar product in $H_{00}^{1 / 2}$. Here, is where the finite element extension theorems play a key role in the discrete case. In Section 4 we present our domain decomposition method which is just the application of iterative methods to the dual problem associated to the Lagrange multipliers. Finally, in Section 5 we show some numerical tests: we consider partitions on two and three subdomains, a four subdomains case with an internal cross point and the case of a floating subdomain, diamond-like shaped with four corners; the results confirm our theoretical analysis. Standard notation, see Girault and Raviart [15] or Adams [1], is used. 


\section{Motivation of the method}

Our model problem is: Given $f \in L^{2}(\Omega)$ we look for $u$ such that

$$
\begin{array}{rll}
-\Delta u+u=f & \text { in } \Omega \\
u=0 & \text { on } \partial \Omega
\end{array}
$$

that has a variational formulation as follows: Given $f \in L^{2}(\Omega)$ we look for $u \in H_{0}^{1}(\Omega)$ such that

$$
(\nabla u, \nabla v)_{\Omega}+(u, v)_{\Omega}=(f, v)_{\Omega} \quad \forall v \in H_{0}^{1}(\Omega)
$$

where $(\varphi, \psi)_{\Omega}=\int_{\Omega} \varphi(x) \psi(x) d x$ is the scalar product in $L^{2}(\Omega)$.

Assume now that $\Omega$ is a polygonal bounded domain in $\mathbb{R}^{2}$ that admits a decomposition without overlapping in polygonal subdomains

$$
\bar{\Omega}=\cup_{r=1}^{R} \bar{\Omega}_{r} \quad \text { and } \quad \Omega_{r} \cap \Omega_{r^{\prime}}=\emptyset, \quad 1 \leq r<r^{\prime} \leq R .
$$

Then, the solution $u$ of (3) also satisfies that

$$
\sum_{r=1}^{R}\left\{\left(\nabla u_{r}, \nabla v_{r}\right)_{\Omega_{r}}+\left(u_{r}, v_{r}\right)_{\Omega_{r}}\right\}=\sum_{r=1}^{R}\left(f_{r}, v_{r}\right)_{\Omega_{r}} \quad \forall v \in H_{0}^{1}(\Omega)
$$

where the subindex $r$ denotes restriction to $\Omega_{r}$, i.e., for instance $u_{r}=u_{\left.\right|_{\Omega_{r}}}$. Next we assume that the partition (4) of $\Omega$ is geometrically conforming in the sense that all interiors interfaces $\Gamma_{r, s}=\overline{\Omega_{r}} \cap \overline{\Omega_{s}} \subset \Omega$ are either a common vertex, a common edge or empty. For simplicity, when $\Gamma_{r, s}$ is an internal common edge we will assume that is a straight open segment without corners. A general case on this situation, i.e., $\Gamma_{r, s}$ with corners, could also be handled in the same way but the description would become more cumbersome. We set $\Gamma_{r, 0}=\partial \Omega_{r} \cap \partial \Omega$ and we may allow $\Gamma_{r, 0}$ polygonal because we impose zero boundary data on $\partial \Omega$. Now we describe $\partial \Omega_{r}$ in terms of its edges via

$$
\partial \Omega_{r}=\Gamma_{r, 0} \cup \Gamma_{r, 1} \cup \ldots \cup \Gamma_{r, J_{r}}
$$

where $J_{r}$ is a positive integer and $\Gamma_{r, 0}$, which might be empty, satisfies

$$
\partial \Omega=\cup_{r=1}^{R} \Gamma_{r, 0} .
$$

We call skeleton of $\bar{\Omega}$, and denote it by $\mathcal{E}$, the set of all interfaces in $\bar{\Omega}$, and by $\mathcal{E}_{0}$ the skeleton of $\Omega$, i.e., the set of all internal interfaces:

$$
\mathcal{E}=\cup_{i=1}^{I} \Gamma_{i}, \quad \mathcal{E}_{0}=\mathcal{E} \cap \Omega=\cup_{i=I_{0}+1}^{I} \Gamma_{i} .
$$


Here $\Gamma_{i}=\Gamma_{i, 0}$ for $i=1, . ., I_{0} \leq R$ describe the boundary $\partial \Omega$, and for $i \geq I_{0}+1$ $\Gamma_{i}=\Gamma_{r, j}$, for some $r, j \geq 1$, are all the internal interfaces.

Then, on each $\Omega_{r}$ we consider the restriction of $H_{0}^{1}(\Omega)$ to $\Omega_{r}$, i.e., the Hilbert space

$$
H_{b}^{1}\left(\Omega_{r}\right)=\left\{v_{r} \in H^{1}\left(\Omega_{r}\right) ; v_{r}=0 \text { on } \partial \Omega_{r} \cap \partial \Omega\right\},
$$

with the classical scalar product $\left(u_{r}, v_{r}\right)_{1, \Omega_{r}}=\left(u_{r}, v_{r}\right)_{\Omega_{r}}+\left(\nabla u_{r}, \nabla v_{r}\right)_{\Omega_{r}}$ and on $\Omega$ the Hilbert space $X$ given by

$X=\left\{v \in L^{2}(\Omega) ; v_{r}=v_{\left.\right|_{\Omega_{r}}} \in H_{b}^{1}\left(\Omega_{r}\right), r \leq R,[v]_{\Gamma_{i}} \in H_{00}^{1 / 2}\left(\Gamma_{i}\right), \quad \forall \Gamma_{i} \in \mathcal{E}_{0}\right\}$

where $[v]_{\Gamma_{i}}$ is the jump across $\Gamma_{i} \in \mathcal{E}_{0}$ given by

$$
[v]_{\Gamma_{i}}=v_{r}-v_{s}, \quad \text { when } \Gamma_{i}=\partial \Omega_{r} \cap \partial \Omega_{s} \subset \Omega \text {. }
$$

The scalar product on $X$ is given by

$$
(u, v)_{X}=\sum_{r=1}^{R}\left(u_{r}, v_{r}\right)_{1, \Omega_{r}}+\sum_{i=I_{0}+1}^{I}\left([u]_{\Gamma_{i}},[v]_{\Gamma_{i}}\right)_{1 / 2,00, \Gamma_{i}}, \forall u, v \in X
$$

and the norm on $X$ is given by

$$
\|v\|_{X}^{2}=\sum_{r=1}^{R}\left\|v_{r}\right\|_{1, \Omega_{r}}^{2}+\sum_{i=I_{0}+1}^{I}\left\|[v]_{\Gamma_{i}}\right\|_{1 / 2,00, \Gamma_{i}}^{2}, \forall v \in X
$$

where we recall (see Grisvard [17] or Adams [1] for instance) that for any open piece of boundary $\Gamma$ of an open and bounded set $\Omega \subset \mathbb{R}^{d}$, the scalar product in $H^{1 / 2}(\Gamma)$ is given for any $v, w \in H^{1 / 2}(\Gamma)$ by

$$
(w, v)_{1 / 2, \Gamma}=\int_{\Gamma} w(x) v(x) d x+\int_{\Gamma} \int_{\Gamma} \frac{(w(x)-w(y))(v(x)-v(y))}{|x-y|^{d}} d x d y
$$

and when $v, w \in H_{00}^{1 / 2}(\Gamma)$ an extra term is added to define the scalar product in $H_{00}^{1 / 2}(\Gamma)$

$$
(w, v)_{1 / 2,00, \Gamma}=(w, v)_{1 / 2, \Gamma}+\int_{\Gamma} \frac{w(x) v(x)}{d(x, \partial \Gamma)} d x
$$


where $d(x, \partial \Gamma)$ is the distance from $x$ to $\partial \Gamma$; then the norm in $H_{00}^{1 / 2}(\Gamma)$ is given by

$$
\|v\|_{1 / 2,00, \Gamma}^{2}=(v, v)_{1 / 2,00, \Gamma} .
$$

The norm on $X$ also measures the jumps across the internal interfaces an thanks to the trace theorems we have the inequality

$$
\|v\|_{X}^{2} \leq C \sum_{r=1}^{R}\left\|v_{r}\right\|_{1, \Omega_{r}}^{2}, \forall v \in X
$$

that will guarantee the ellipticity of the problems that will be posed later on.

We can identify the space $H_{0}^{1}(\Omega)$ with the subspace $V$ of elements of $X$ such that their jumps are zero on the interfaces. Then, the unique solution $u$ of our variational problem (3) also solves the problem:

Find $u \in V$ such that for all $v \in V$

$$
\sum_{r=1}^{R}\left\{\left(\nabla u_{r}, \nabla v_{r}\right)_{\Omega_{r}}+\left(u_{r}, v_{r}\right)_{\Omega_{r}}\right\}=\sum_{r=1}^{R}\left(f_{\left.\right|_{\Omega_{r}}}, v_{r}\right)_{\Omega} .
$$

Our purpose now is to get rid of the constrains on the jumps and set (8) on $X$. This will be achieved by adding the restriction on the jumps via Lagrangian multipliers to (8). Therefore we must characterize $H_{0}^{1}(\Omega)$ in $X$.

\subsection{Description of $H_{0}^{1}(\Omega)$ within $X$}

To achieve this description we follow the idea introduced by Raviart-Thomas [22] and study the linear forms on $X$ that vanish on $H_{0}^{1}(\Omega)$. We must guarantee that all the jumps across internal interfaces vanish and we do this via Lagrangian multipliers.

A key ingredient is the Green formula on polygonal domains and the localization of the boundary integrals on each element $\Gamma_{i} \in \mathcal{E}_{0}$ so as to act on the jumps. It is in this point where we improve the arguments in [22]. As a reward, our analysis will say that cross points do not matter in the computation of the solution and the characterization in [22] will be improved.

Let $\mathcal{O}$ be a polygonal domain in $\mathbb{R}^{2}$ with edges $\Gamma_{j}, 1 \leq j \leq J$. The domain of the divergence operator on $\mathcal{O}$ is

$$
H(\operatorname{div} ; \mathcal{O})=\left\{\vec{q} \in L^{2}(\mathcal{O})^{2} ; \operatorname{div}(\vec{q}) \in L^{2}(\mathcal{O})\right\}
$$


For each $j$, we also introduce the space

$$
H_{(j)}^{1}(\mathcal{O})=\left\{v \in H^{1}(\mathcal{O}) ; v=0 \text { on } \partial \mathcal{O} \backslash \bar{\Gamma}_{j}\right\},
$$

and we recall that the trace operator: $v \mapsto v_{\mid \Gamma_{j}}$ is continuous from $H_{(j)}^{1}(\mathcal{O})$ onto $H_{00}^{1 / 2}\left(\Gamma_{j}\right)$. The next result is then easily derived from the density of $\mathcal{D}(\overline{\mathcal{O}})^{2}$ into $H(d i v ; \mathcal{O})$, see [15], Chap. I, Thm 2.4 for instance. We refer to Grisvard [17] for a more detailed proof in the case of a polygon.

Let $\langle\cdot, \cdot\rangle_{-1 / 2,00, \Gamma_{j}}$ denote the duality $H_{00}^{-1 / 2}\left(\Gamma_{j}\right)-H_{00}^{1 / 2}\left(\Gamma_{j}\right)$, then

Lemma 1 The normal trace operator defined by

$$
\forall v \in H_{(j)}^{1}(\mathcal{O}), \quad<\mathbf{n}_{j} \cdot \vec{q}, v>_{-1 / 2,00, \Gamma_{j}}=(\vec{q}, \nabla v)_{\mathcal{O}}+(\operatorname{div}(\vec{q}), v)_{\mathcal{O}},
$$

admits a continuous extension from $H($ div $; \mathcal{O})$ into the dual space $H_{00}^{-1 / 2}\left(\Gamma_{j}\right)$ of $H_{00}^{1 / 2}\left(\Gamma_{j}\right)$.

Let also $\mathrm{E}$ be the space

$$
E=\left\{v \in H^{1}(\mathcal{O}) ; \Delta v \in L^{2}(\mathcal{O})\right\} .
$$

The next corollary is derived by applying Lemma 1 to the functions $\nabla v$ when $v \in E$.

Corollary 1 The normal derivative defined by

$$
\forall v \in H_{(j)}^{1}(\mathcal{O}), \quad<\partial_{\mathbf{n}_{j}} u, v>_{-1 / 2,00, \Gamma_{j}}=(\nabla u, \nabla v)_{\mathcal{O}}+(\Delta u, v)_{\mathcal{O}},
$$

admits a continuous extension from $E$ into the space $H_{00}^{-1 / 2}\left(\Gamma_{j}\right)$.

As a consequence, we have the following integrations by parts

Corollary 2 When $\mathcal{O} \subset \mathbb{R}^{2}$ is a polygonal domain and $\partial \mathcal{O}=\cup_{j=1}^{J} \Gamma_{j}$, then for each $u \in E$ and any $\vec{q} \in H($ div $; \mathcal{O})$ we have

$$
\begin{aligned}
(\Delta u, v)_{\mathcal{O}}+(\nabla u, \nabla v)_{\mathcal{O}} & =\sum_{j=1}^{J}<\partial_{\mathbf{n}_{j}} u, v>_{-1 / 2,00, \Gamma_{j}} \\
(\vec{q}, \nabla v)_{\mathcal{O}}+(\operatorname{div}(\vec{q}), v)_{\mathcal{O}} & =\sum_{j=1}^{J}<\mathbf{n}_{j} \cdot \vec{q}, v>_{-1 / 2,00, \Gamma_{j}}
\end{aligned}
$$

for any $v \in H^{1}(\mathcal{O})$ with $v_{\left.\right|_{\Gamma_{j}}} \in H_{00}^{1 / 2}\left(\Gamma_{j}\right)$ for $j=1,2, \ldots, J$. 
As each $\Omega_{r}$ is a polygonal domain, and as a consequence of the above results, we need to consider the dense subspace $W_{r}$ of $H_{b}^{1}\left(\Omega_{r}\right)$ given by, observe that we consider only internal interfaces,

$$
W_{r}=\left\{u \in H_{b}^{1}\left(\Omega_{r}\right) ; u_{\Gamma_{r, j}} \in H_{00}^{1 / 2}\left(\Gamma_{r, j}\right), j=1, \ldots, J_{r}\right\},
$$

and the dense subspace in $X$ given by

$$
X_{0}=\left\{v \in L^{2}(\Omega) ; v_{r}=v_{\Omega_{\Omega_{r}}} \in W_{r}, r=1, \ldots, R\right\} .
$$

The use of $X_{0}$ is a key tool in our analysis because the Green's formula on polygonal subdomains can be applied on each $\Omega_{r}$. Now, as we are only interested in what happens on the internal interfaces, we consider

$$
M=\left\{\vec{\mu} \in \prod_{r=1}^{R} \prod_{j=1}^{J_{r}} H_{00}^{-1 / 2}\left(\Gamma_{r, j}\right) ; \mu_{r, j}=\mathbf{n}_{r, j} \cdot \vec{q}, \text { for some } \vec{q} \in H(\operatorname{div} ; \Omega) .\right\}
$$

The elements of $M$ will be denoted the Lagrange multipliers on the internal interfaces $\Gamma_{i} \in \mathcal{E}_{0}$. The following result gives a description of the linear forms in $X$ that vanish on $H_{0}^{1}(\Omega)$ :

Lemma $2 L \in X^{\star}$ (dual space of $X$ ) vanishes on $H_{0}^{1}(\Omega)$ if and only if there exists a unique $\vec{\mu} \in M$ such that

$$
L(u)=\sum_{r=1}^{R} \sum_{j=1}^{J_{r}}<\mu_{r, j}, u_{r}>_{-1 / 2,00, \Gamma_{r, j}}, \quad \forall u \in X_{0} .
$$

Observation 1 Using $\vec{q} \in H($ div $; \Omega)$ such that $\left(\mathbf{n}_{t, s} \cdot \vec{q}\right)=\mu_{t, s}$, the fact that $\mathbf{n}_{s, t}=-\mathbf{n}_{t, s}$ on any internal interface $\Gamma_{i}=\overline{\Omega_{s}} \cap \overline{\Omega_{t}}$ and a density argument, (11) can be replaced by (12)

$$
L(u)=\sum_{i=I_{0}+1}^{I}<\mu_{i},[u]_{\Gamma_{i}}>_{-1 / 2,00, \Gamma_{i}}, \quad \forall u \in X
$$

where we recall that $\Gamma_{i}$ for $i \geq I_{0}+1$ are the internal interfaces, i.e., we only act on the jumps across internal interfaces.

Dem: We give the main idea and the rest is left to reader. For any $L \in X^{\star}$ via Riesz representation there exists a unique $v \in X$ such that $L(u)=(v, u)_{X}$ 
for all $u, v \in X$. Then, for each $u_{r} \in H_{0}^{1}\left(\Omega_{r}\right)$ let $\tilde{u}_{r}$ be its extension by zero to $H_{0}^{1}(\Omega)$, then $\tilde{u}_{r} \in X$ and each $v_{r} \in H_{b}^{1}\left(\Omega_{r}\right)$ satisfies

$$
(\tilde{u}, v)_{X}=\left(u_{r}, v_{r}\right)_{1, \Omega_{r}}=\left(u_{r}, v_{r}\right)_{\Omega_{r}}+\left(\nabla u_{r}, \nabla v_{r}\right)_{\Omega_{r}}=0 \quad \forall u_{r} \in H_{0}^{1}\left(\Omega_{r}\right)
$$

which implies that $v_{r} \in H_{b}^{1}\left(\Omega_{r}\right)$ and $\Delta v_{r}=v_{r} \in L^{2}\left(\Omega_{r}\right)$. Now recall that $\partial \Omega_{r}=\cup_{j=0}^{J_{r}} \Gamma_{r, j}$, then $\partial_{\mathbf{n}_{r, j}} v_{r} \in H_{00}^{-1 / 2}\left(\Gamma_{r, j}\right)$ where $\mathbf{n}_{r, j}$ is the outward vector normal on $\Gamma_{r, j}$. As we can not deduce more regularity on $v_{r}$ we must apply Green formula on polygonal domains with functions in $W_{r}$ and then (observe that $\Gamma_{r, 0}=\partial \Omega_{r} \cap \partial \Omega$ does not appear because $u_{r}=0$ on $\left.\Gamma_{r, 0}\right)$

$$
\left(u_{r}, v_{r}\right)_{1, \Omega_{r}}=\sum_{j=1}^{J_{r}}<\partial_{\mathbf{n}_{r, j}} v_{r}, u_{r}>_{-1 / 2,00, \Gamma_{r, j}} \quad \forall u_{r} \in W_{r},
$$

which implies that for all $u \in X_{0}$

$$
L(u)=(v, u)_{X}=\sum_{r=1}^{R} \sum_{j=1}^{J_{r}}<\partial_{\mathbf{n}_{r, j}} v_{r}, u_{r}>_{-1 / 2,00, \Gamma_{r, j}}
$$

where again observe that only the internal interfaces $\left(\Gamma_{r, j}\right.$ with $\left.j \geq 1\right)$ are considered. A key observation is that all these normal derivatives are related because the fact that $L(u)=0$ for all $u \in H_{0}^{1}(\Omega)$ implies

$$
(u, s)_{\Omega}+(\nabla u, \vec{q})_{\Omega}=0 \quad \forall u \in H_{0}^{1}(\Omega)
$$

where $\vec{q} \in L^{2}(\Omega)^{d}$ and $s \in L^{2}(\Omega)$ are given locally by

$$
\vec{q}_{\Omega_{r}}=\nabla v_{r}\left(\Rightarrow \mathbf{n}_{r, j} \cdot \vec{q}=\partial_{\mathbf{n}_{r, j}} v_{r}\right), \quad s_{\left.\right|_{\Omega_{r}}}=v_{r}=\Delta v_{r}
$$

Then, (13) tells that $\vec{q} \in H(\operatorname{div} ; \Omega)$ and $\operatorname{div}(\vec{q})=s \in L^{2}(\Omega)$ (no information is obtained for $\mathbf{n}_{r, 0} \cdot \vec{q}_{r} \in H_{00}^{-1 / 2}\left(\Gamma_{r, j}\right)$, but it is not needed). Finally, thanks to this $\vec{q}$ that relates the normal derivatives across common interfaces we can write, using $\mu_{i}=\mathbf{n}_{r, j} \cdot \vec{q}=-\mathbf{n}_{j, r} \cdot \vec{q}$ on $\Gamma_{i}=\Gamma_{r, j}$, that

$$
L(u)=\sum_{i=I_{0}+1}^{I}<\mu_{i},[u]_{\Gamma_{i}}>_{-1 / 2,00, \Gamma_{i}}, \quad \forall u \in X_{0},
$$

i.e., we only act on the jumps across internal interfaces. The extension of this last expresion for $L$ when acting on $X_{0}$ to $X$ by a density argument is simple.

As a consequence, we have the characterization of $H_{0}^{1}(\Omega)$ as a subspace of $X$ given by 
Lemma 3 Let $b: M \times X \mapsto \mathbb{R}$ be defined for $v \in X$ and $\vec{\lambda} \in M$ by

$$
b(\vec{\lambda}, v)=\sum_{i=I_{0}+1}^{I}<\lambda_{i},[v]_{\Gamma_{i}}>_{-1 / 2,00, \Gamma_{i}} .
$$

Then

$$
H_{0}^{1}(\Omega)=\{v \in X ; b(\vec{\lambda}, v)=0, \quad \forall \vec{\lambda} \in M\}
$$

Dem: We just prove that $B=\{v \in X ; b(\vec{\lambda}, v)=0, \quad \forall \vec{\lambda} \in M\} \subset H_{0}^{1}(\Omega)$ because it is clear the inclusion $H_{0}^{1}(\Omega) \subset B$. As $X=H_{0}^{1}(\Omega) \oplus H_{0}^{1}(\Omega)^{\perp, X}$, where $\oplus$ stands for orthogonal sum, for any $v \in B$ we have $v=v_{0}+v_{1}$ with $v_{0} \in H_{0}^{1}(\Omega)$ and $v_{1} \in H_{0}^{1}(\Omega)^{\perp, X}$. Take then the linear form $T_{v_{1}}$ on $X$ associated with $v_{1}$ via Riesz representation

$$
T_{v_{1}}(u)=\left(v_{1}, u\right)_{X}, \quad \forall u \in X
$$

As $T_{v_{1}}$ vanishes on $H_{0}^{1}(\Omega)$ there exists a unique $\vec{\mu} \in M$ such that for all $u \in X$

$$
\left(v_{1}, u\right)_{X}=b(\vec{\mu}, u)=\sum_{i=I_{0}+1}^{I}<\mu_{i},\left[u_{i}\right]_{\Gamma_{i}}>_{-1 / 2,00, \Gamma_{i}} .
$$

Then, using (15) and that $v \in B$

$$
\left\|v_{1}\right\|_{X}^{2}=\left(v_{1}, v_{1}\right)_{X}=\left(v_{1}, v\right)_{X}=b(\vec{\mu}, v)=0
$$

then $v_{1}=0$ and $v=v_{0} \in H_{0}^{1}(\Omega)$.

\subsection{Lagrange formulation or primal hybrid formula- tion of the model problem}

Define the bilinear form $a: X \times X \mapsto \mathbb{R}$ given by

$$
a(u, v)=(u, v)_{X}=\sum_{r=1}^{R}\left(u_{r}, v_{r}\right)_{1, \Omega_{r}}=\sum_{r=1}^{R} \int_{\Omega_{r}}\left\{\nabla u_{r} \cdot \nabla v_{r}+u_{r} v_{r}\right\} d x .
$$

Thanks to the trace inequalities $(7)$ the ellipticity of $a(\cdot, \cdot)$ on $X$ is clear. We also use the bilinear form $b(\vec{\lambda}, v)$ given in (14). Then, our Dirichlet problem 
(1) consists in looking for a pair $(u, \vec{\lambda}) \in X \times M$ such that

$$
\begin{aligned}
a(u, v)+b(\vec{\lambda}, v) & =\sum_{r=1}^{R}\left(f, v_{r}\right)_{\Omega_{r}}, \forall v \in X \\
b(\vec{\mu}, u) & =0, \quad \forall \vec{\mu} \in M .
\end{aligned}
$$

This formulation is also known as a primal hybrid formulation because it mixes the primal variable $u$ with the Lagrangian multipliers that constraint the jumps. We have the equivalence result

Theorem $3 u \in H_{0}^{1}(\Omega)$ solves the Dirichlet problem (3) if and only if there exists a unique $\vec{\lambda} \in M$ such that $(u, \vec{\lambda}) \in X \times M$ solves problem (17)-(18). Moreover, in this case and for $i=I_{0}+1, \ldots, I$,

$$
\lambda_{i}=-\partial_{\mathbf{n}_{i}} u \in H_{00}^{-1 / 2}\left(\Gamma_{i}\right) .
$$

Dem: Let $(u, \vec{\lambda}) \in X \times M$ solve (17)-(18). Then, $u \in H_{0}^{1}(\Omega)$ due to (18) and for any $v \in H_{0}^{1}(\Omega) \subset X$

$$
(\nabla u, \nabla v)_{\Omega}+(u, v)_{\Omega}=(f, v)_{\Omega}, \quad \forall v \in H_{0}^{1}(\Omega)
$$

which is (3). On the other hand, if $u \in H_{0}^{1}(\Omega)$ solves (3) then (18) holds and the mapping $T_{u} \in X^{\star}$ given by

$$
T_{u}(v)=(f, v)_{\Omega}-a(u, v) \quad \forall v \in X
$$

vanishes on $H_{0}^{1}(\Omega)$. Therefore, there exists a unique $\vec{\lambda} \in M$ such that (17)

$$
(f, v)_{\Omega}-a(u, v)=\sum_{i=I_{0}+1}^{I}<\lambda_{i},[v]_{\Gamma_{i}}>_{-1 / 2,00, \Gamma_{i}} \quad \forall v \in X
$$

holds. Moreover, (19) holds by using that $f=-\Delta u+u$ and integrating by parts with elements on $X_{0}$. 


\section{Formulation using Riesz representation}

The cornerstone now is how to compute the dualities that act on the jumps. This question has been considered from several points of view like mesh dependent scalar products (Barbosa-Hughes [3]), wavelet bases (Bertoluzza [5]) or projection operators onto the $L^{2}$ space on the interfaces (Ben Belgacem [4]). In our approach we use Riesz representation and work with the $H_{00}^{1 / 2}$ scalar product that is explicitly computed.

Via Riesz representation we identify $H_{00}^{-1 / 2}\left(\Gamma_{i}\right)$ (dual space of $H_{00}^{1 / 2}\left(\Gamma_{i}\right)$ ) with $H_{00}^{1 / 2}\left(\Gamma_{i}\right)$ and therefore identify $M$ with its dual space $M^{\star}$. Then we write all the dualities in terms of the scalar product in $H_{00}^{1 / 2}\left(\Gamma_{i}\right)$. To simplify notation, we denote the elements of $H_{00}^{1 / 2}\left(\Gamma_{i}\right)$ likewise those of $H_{00}^{-1 / 2}\left(\Gamma_{i}\right)$, define the continuous bilinear form $b: M \times X \mapsto \mathbb{R}$ given by

$$
b(\vec{\lambda}, v)=\sum_{i=I_{0}+1}^{I}\left(\lambda_{i},[v]_{\Gamma_{i}}\right)_{-1 / 2,00, \Gamma_{i}} .
$$

Then, the formulation of Poisson problem (3) that we shall use is: Find a pair $(u, \vec{\lambda}) \in X \times M$ such that

$$
\begin{aligned}
a(u, v)+b(v, \vec{\lambda}) & =\sum_{r=1}^{R}\left(f, v_{r}\right)_{\Omega_{r}}, \forall v \in X, \\
b(u, \vec{\mu}) & =0, \forall \vec{\mu} \in M .
\end{aligned}
$$

where we recall that the bilinear form $a: X \times X \mapsto \mathbb{R}$ is given by (16). Thanks to Theorem 3 formulation (22)-(23) is equivalent to (3) but with the difference that now each $\lambda_{i}$ is the Riesz representation of the normal derivative $-\partial_{\mathbf{n}_{i}} u \in H_{00}^{-1 / 2}\left(\Gamma_{i}\right)$.

Moreover, problem (22)-(23) is whithin the saddle point problems framework, see Girault-Raviart [15] Chap. I, Section 4.2. In fact, thanks to the finite element extension theorems, see for instance Bernardi-Maday-Rapetti [7], Section IX.4, we can handle at the same time the infinite dimensional and the finite dimensional versions of (22)-(23) as follows: for a conforming triangulation $\mathcal{T}_{h}$ of $\Omega$ that contains the skeleton $\mathcal{E}$ as union of edges of triangles, we consider a family of Lagrange finite element subspaces $\left\{X_{h}\right\}_{h} \subset X$, where $h$ is the discretization parameter, and the family $\left\{M_{h}\right\}_{h} \subset M$ of their restrictions, or traces, to the interfaces, $M_{h}=\left(X_{h}\right)_{\left.\right|_{\mathcal{E}}}$. Then, we pose equations (22)-(23) on $X$ and $M$ but also on $X_{h} \subset X$ and $M_{h} \subset M$. For these 
pairs of families of finite element spaces $\left\{X_{h}\right\}_{h}$ and $\left\{M_{h}\right\}_{h}$ the finite element extension theorems apply. This fact will allow the uniform, with respect to $h$, version of the discrete inf-sup condition. As a consequence, we can use any iterative method, like the method of Uzawa or Conjugate Gradient method, which is at the basis of FETI methods, to compute the solution, with condition numbers of the iterative matrices independent of the mesh size $h$.

First, we see that the ellipticity of $a$ on $X$ or $X_{h}$ is straightforward because of the $(7)$. Second, the bilinear form $b(\cdot, \cdot)$ satisfies inf-sup conditions with constants that are independent of $h$ on the pairs $X \times M$ and $X_{h} \times M_{h}$ thanks to the finite element extension theorems:

Theorem 4 Assume that the family of triangulations $\left\{\mathcal{T}_{h}\right\}_{h}$ is regular, then there exist positive constants $\alpha, \beta>0$ such that the bilinear form $b(\cdot, \cdot)$ : $M \times X \mapsto \mathbb{R}$ given by (21) is continuous and satisfies an inf-sup condition, i.e.,

$$
\sup _{\vec{\mu} \in M} \sup _{v \in X} \frac{b(\vec{\mu}, v)}{\|\vec{\mu}\|_{M}\|v\|_{X}}=\alpha, \quad \inf _{\vec{\mu} \in M} \sup _{v \in X} \frac{b(\vec{\mu}, v)}{\|\vec{\mu}\|_{M}\|v\|_{X}} \geq \beta .
$$

These expression also hold in the discrete case independently of $h$ : the restriction of the bilinear form $b(\cdot, \cdot)$ given by (21) to the subspace $M_{h} \times X_{h}$ is also continuous and satisfies an inf-sup condition uniform in $h$, i.e.,

$$
\sup _{\vec{\mu} \in M_{h}} \sup _{v \in X_{h}} \frac{b(\vec{\mu}, v)}{\|\vec{\mu}\|_{M}\|v\|_{X}}=\alpha, \quad \inf _{\vec{\mu} \in M_{h}} \sup _{v \in X_{h}} \frac{b(\vec{\mu}, v)}{\|\vec{\mu}\|_{M}\|v\|_{X}}=\beta>0 .
$$

Proof: First bound in (24) or (25) is due to the continuity of the trace operator. Now we prove the second bound in (25), i.e., the inf-sup condition in the discrete case. Take $\mu_{h} \in M$ and set $\mu_{i, h} \in H_{00}^{1 / 2}\left(\Gamma_{i}\right)$ for any $\Gamma_{i} \in \mathcal{E}_{0}$. Let us suppose that $\Gamma_{i} \in \partial \Omega_{r}$ for some $r$; then as $\mu_{i, h} \in H_{00}^{1 / 2}\left(\Gamma_{i}\right)$ is a continuous piecewise polynomial function there exists a discrete extension $E_{r, h} \mu_{i, h} \in\left(X_{h}\right)_{\Omega_{\Omega_{r}}}$ to the subdomain $\Omega_{r}$ such that $E_{r, h} \mu_{i, h}=0$ on $\partial \Omega_{r} \backslash \Gamma_{i}$ and there exists a positive constant $\gamma>0$ independent of $h$ such that

$$
\left\|E_{r, h} \mu_{i, h}\right\|_{1, \Omega_{r}} \leq \gamma^{-1}\left\|\mu_{i, h}\right\|_{1 / 2,00, \partial \Omega_{r}}=\gamma^{-1}\left\|\mu_{i, h}\right\|_{1 / 2,00, \Gamma_{i}}
$$

these results are a simple consequence of the finite element extension theorems, see [7] Th. IX.4.1. Take now $\widetilde{E}_{r, h} \mu_{i, h} \in X_{h}$ the extension by zero of $E_{r, h} \mu_{i, h}$ from $\Omega_{r}$ to the whole of $\Omega$, then

$$
\left[\widetilde{E}_{r, h} \mu_{i, h}\right]_{\Gamma_{i}}=\mu_{i, h}, \quad \text { and } \quad\left[\widetilde{E}_{r, h} \mu_{i, h}\right]_{\Gamma_{j}}=0, \quad j \neq i,
$$


that implies

$$
\left(\mu_{j},\left[\widetilde{E}_{r, h} \mu_{i, h}\right]\right)_{-1 / 2,00, \Gamma_{j}}=0, \quad j \neq i,
$$

and also

$$
\left\|\widetilde{E}_{r, h} \mu_{i, h}\right\|_{1, \Omega}=\left\|E_{r, h} \mu_{i, h}\right\|_{1, \Omega_{r}} \leq \gamma^{-1}\left\|\mu_{i, h}\right\|_{1 / 2,00, \Gamma_{i}} .
$$

As a consequence, for any given $\mu_{h} \in M_{h}$, we consider $v^{\mu_{h}} \in X_{h}$ given by $v^{\mu_{h}}=\sum_{i=I_{0}+1}^{I} \widetilde{E}_{r, h} \mu_{i, h}$. Then

$$
\left\|v^{\mu_{h}}\right\|_{X} \leq \sqrt{I_{1}} \gamma^{-1}\left\|\mu_{h}\right\|_{M}
$$

where $I_{1}$ is the number of internal interfaces, and finally

$$
\begin{aligned}
b\left(\mu_{h}, v^{\mu_{h}}\right) & =\sum_{i=I_{0}+1}^{I}\left(\mu_{i, h},\left[\widetilde{E}_{r, h} \mu_{i, h}\right]\right)_{-1 / 2,00, \Gamma_{i}}=\sum_{i=I_{0}+1}^{I}\left\|\mu_{i, h}\right\|_{1 / 2,00, \Gamma_{i}}^{2} \\
& \geq \beta\left\|v^{\mu_{h}}\right\|_{X}\left\|\mu_{h}\right\|_{1 / 2,00, \Gamma}
\end{aligned}
$$

for $\beta=\gamma I_{1}^{-1 / 2}$. Therefore, the discrete inf-sup condition holds uniformly in $h$.

Next, we prove the second bound in (24), i.e., the inf-sup condition in the continuous case. An elegant proof is obtained when we consider the multipliers $\lambda_{i}$ as elements in $H_{00}^{-1 / 2}\left(\Gamma_{i}\right)$. We know that $\vec{\lambda}=\left(\lambda_{i}\right)_{i=I_{0}+1}^{I}$ where $\Gamma_{i}$ are the internal interfaces and

$$
\|\vec{\lambda}\|_{M}^{2}=\sum_{r=1}^{R} \sum_{j=1}^{J_{r}}\left\|\lambda_{r, j}\right\|_{-1 / 2,00, \Gamma_{r, j}}^{2}=2 \sum_{i=I_{0}+1}^{I}\left\|\lambda_{i}\right\|_{-1 / 2,00, \Gamma_{i}}^{2} .
$$

For any $\Omega_{r}$ we denote by $I_{r}$ the set of indices $i$ such that $\partial \Omega_{r} \cap \Omega=\cup_{i \in I_{r}} \Gamma_{i}$. Then, for any $i \in I_{r}$ there exists $w_{i} \in W_{r}$ such that

$$
\begin{aligned}
-\Delta w_{i}+w_{i} & =0 \text { en } \Omega_{r} \\
w_{i} & =0 \text { en } \partial \Omega_{r} \backslash \Gamma_{i} \\
\partial_{n_{i}} w_{i} & =\lambda_{i} \in H_{00}^{-1 / 2}\left(\Gamma_{i}\right)
\end{aligned}
$$

and as a consequence for all $v \in W_{r}$ with $v=0$ on $\Gamma_{j}$ for $i, j \in I_{r}$ with $j \neq i$ we have

$$
\left(w_{i}, v\right)_{1, \Omega_{r}}=<\lambda_{i}, v>_{-1 / 2,00, \Gamma_{i}} \Rightarrow\left\|w_{i}\right\|_{1, \Omega_{r}}^{2}=\left\|\lambda_{i}\right\|_{-1 / 2,00, \Gamma_{i}}^{2} .
$$


Then, $w_{r}=\sum_{i \in I_{r}} w_{i} \in W_{r} \subset H_{b}^{1}\left(\Omega_{r}\right)$ satisfies

$$
\left\|w_{r}\right\|_{1, \Omega_{r}}^{2}=\sum_{i \in I_{r}}\left\|w_{i}\right\|_{1, \Omega_{r}}^{2}=\sum_{i \in I_{r}}\left\|\lambda_{i}\right\|_{-1 / 2,00, \Gamma_{i}}^{2}
$$

because $\left(w_{i}, w_{j}\right)_{1, \Omega_{r}}=0$ when $i, j \in I_{r}$ with $j \neq i$. Set now $w_{\star} \in X_{0}$ given by $w_{\star}=w_{r}$ on $\Omega_{r}$, it holds that

$$
\sum_{r=1}^{R}\left\|w_{r}\right\|_{1, \Omega_{r}}^{2}=\sum_{r=1}^{R} \sum_{i \in I_{r}}\left\|w_{i}\right\|_{1, \Omega_{r}}^{2}=\sum_{r=1}^{R} \sum_{i \in I_{r}}\left\|\lambda_{i}\right\|_{-1 / 2,00, \Gamma_{i}}^{2}=\|\vec{\lambda}\|_{M}^{2}
$$

and therefore, using (7),

$$
\begin{aligned}
b\left(\vec{\lambda}, w_{\star}\right) & =\sum_{r=1}^{R} \sum_{i=1}^{I_{r}}<\lambda_{i}, w_{i}>_{-1 / 2,00, \Gamma_{i}}=\sum_{r=1}^{R}\left(w_{r}, w_{r}\right)_{1, \Omega_{r}} \\
& =\sum_{r=1}^{R}\left\|w_{r}\right\|_{1, \Omega_{r}}^{2} \geq C^{-1}\left\|w_{\star}\right\|_{X}\|\vec{\lambda}\|_{M} .
\end{aligned}
$$




\section{Domain decomposition method}

Our saddle point problem can be written as equations in $X^{\star} \times M$ : find $(u, \lambda) \in X \times M$ such that

$$
\begin{array}{rlc}
R^{-1} u+B^{\star} \lambda & =F \quad \text { on } X^{\star} \\
B u & =0 \quad \text { on } M,
\end{array}
$$

where $R: X^{\star} \mapsto X$ is the Riesz isomorphism associated with the bilinear form $a(\cdot, \cdot)$ and defined by

$$
<R^{-1} u, v>=a(u, v), \quad \forall u, v \in X,
$$

$B$ is the continuous mapping $B: X \mapsto M$ defined by

$$
B v=\left([v]_{\Gamma_{i}}\right)_{i=I_{0}+1}^{I},
$$

i.e., $B v$ gives the jumps across the internal interfaces $\Gamma_{i} \in \mathcal{E}_{0}$ of $v$ and $B^{\star}$ is the transpose operator to $B$. Then

$$
b(\mu, v)=\sum_{i=I_{0}+1}^{I}\left(\mu_{i},[v]_{\Gamma_{i}}\right)_{1 / 2,00, \Gamma_{i}}=(\mu, B v)_{M} \quad \forall v \in X .
$$

Finally, we take $F: X \mapsto \mathbb{R}$ given by

$$
<F, v>=\sum_{r=1}^{2}\left(f, v_{r}\right)_{\Omega_{r}}=(f, v)_{\Omega} .
$$

As a consequence,

$$
u=R\left(F-B^{\star} \lambda\right) \Rightarrow B u=B R F-B R B^{\star} \lambda
$$

and using $B u=0$ from here we have the dual problem associated to the saddle point problem

$$
\left(B R B^{\star}\right) \lambda=B R F \text { on } M
$$

Thanks to the inf-sup condition, on the infinite dimensional or finite dimensional setting, the operator $B R B^{\star}$ is symmetric positive definite with eigenvalues in the interval $\left[\beta^{2}, \alpha^{2}\right]$ where $\beta^{2}, \alpha^{2}>0$ are independent of 
the discretization parameter $h$; it also holds that $\beta^{2}, \alpha^{2}$ are eigenvalues of $B R B^{\star}$. Moreover, the energy norm on $M$ associated to the operator $B R B^{\star}$ is a equivalent norm on $M$ that satisfies

$$
\beta^{2}\|\lambda\|_{1 / 2,00, \Gamma}^{2} \leq\left(B R B^{\star} \lambda, \lambda\right)_{1 / 2,00, \Gamma} \leq \alpha^{2}\|\lambda\|_{1 / 2,00, \Gamma}^{2}, \quad \forall \lambda \in M,
$$

see for instance Bacuta [2]. As a consequence, the condition number of the operator $B R B^{\star}$ is bounded independently of the discretization parameter,

$$
\kappa=\kappa\left(B R B^{\star}\right) \leq \frac{\alpha^{2}}{\beta^{2}} .
$$

This result improves the estimate given by Mandel-Tezaur [19] where the estimate on the condition number is expressed asymptotically by

$$
C(1+\log (H / h))^{2}
$$

where $H$ and $h$ are the characteristic subdomain size and element size respectively.

Now the resolution of (29) via an iterative method is possible. We propose the use of the iterative method of Richardson, which amounts to the classical method of Uzawa and also the Conjugate Gradient Method. Both yield non overlapping domain decomposition iteration techniques and the second one is the basics of the standard FETI method. We also observe that Conjugate Gradient method does not need any preconditioning.

\subsection{Uzawa and Conjugate Gradient methods}

The classical iteration method of Uzawa is: Given $\rho>0$ and $\lambda_{0} \in M$, for $m=0,1,2,3, \ldots$ set

$$
\begin{aligned}
r_{m} & =B R F-\left(B R B^{\star}\right) \lambda_{m}=B u_{m}, \text { using (28) } \\
\lambda_{m+1} & =\lambda_{m}+\rho r_{m} .
\end{aligned}
$$

To fix ideas we consider the case where we split $\Omega$ slicewise into two subdomains $\Omega_{1}, \Omega_{2}$ and we assume that $\Gamma=\partial \Omega_{1} \cap \partial \Omega_{2}$ is a straight segment. Then we have $M=H_{00}^{1 / 2}(\Gamma), X_{0}=X$ and our formulation is:

Find a pair $(u, \lambda) \in X \times M$ such that for all $v \in X$ and $\mu \in M$

$$
\begin{aligned}
\sum_{r=1}^{2}\left\{\left(\nabla u_{r}, \nabla v_{r}\right)_{\Omega_{r}}+\left(u_{r}, v_{r}\right)_{\Omega_{r}}\right\}+\left(\lambda, v_{1}-v_{2}\right)_{1 / 2,00, \Gamma} & =\sum_{r=1}^{2}\left(f, v_{r}\right)_{\Omega_{r}}(32 \\
\left(\mu, u_{1}-u_{2}\right)_{1 / 2,00, \Gamma} & =0
\end{aligned}
$$


Then, Uzawas' method unfolds from (32)-(33) as:

Given $\rho>0$ and $\lambda_{0} \in M$, for $m \geq 0$ find $u_{m} \in X$ such that for all $v \in X$

$$
\begin{aligned}
& \qquad \begin{aligned}
\sum_{r=1}^{2}\left(u_{m, r}, v_{r}\right)_{1, \Omega_{r}} & =\sum_{r=1}^{2}\left(f, v_{r}\right)_{\Omega_{r}}-\left(\lambda_{m}, v_{1}-v_{2},\right)_{1 / 2,00, \Gamma}, \\
\text { and update } \lambda_{m+1} & =\lambda_{m}+\rho\left(u_{m, 1}-u_{m, 2}\right) .
\end{aligned}
\end{aligned}
$$

where recall that $\left(u_{m, r}, v_{r}\right)_{1, \Omega_{r}}=\left(\nabla u_{r}, \nabla v_{r}\right)_{\Omega_{r}}+\left(u_{r}, v_{r}\right)_{\Omega_{r}}$. We have geometric convergence for this iterative process, see Bacuta [2] and references therein:

Theorem 5 For $\left.\rho \in] 0,2 \alpha^{-2}\right]$ and any $\lambda_{0} \in H_{00}^{1 / 2}(\Gamma)$, the iteration process (34)-(35) converges geometrically to the solution of Poisson problem. More precisely, as the the operator $B R B^{\star}$ is symmetric positive definite with eigenvalues in the interval $\left[\beta^{2}, \alpha^{2}\right]$ we have

$$
\begin{aligned}
\left\|u_{m}-u\right\|_{X} & \leq \alpha\left\|\lambda_{m}-\lambda\right\|_{1 / 2,00, \Gamma} \\
\left\|\lambda_{m+1}-\lambda\right\|_{1 / 2,00, \Gamma} & \leq \max \left\{\left|1-\rho \beta^{2}\right|,\left|1-\rho \alpha^{2}\right|\right\}\left\|\lambda_{m}-\lambda\right\|_{1 / 2,00, \Gamma}
\end{aligned}
$$

The optimal convergence factor is achieved for $\rho_{\text {opt }}=2 /\left(\alpha^{2}+\beta^{2}\right)$ and in this case, for $m \geq 0$, we have

$$
\left\|\lambda_{m+1}-\lambda\right\|_{1 / 2,00, \Gamma} \leq \frac{\kappa^{2}-1}{\kappa^{2}+1}\left\|\lambda_{m}-\lambda\right\|_{1 / 2,00, \Gamma} .
$$

where $\kappa=\alpha^{2} / \beta^{2}$ is the spectral condition number of $B R B^{\star}$ that is independent of the discretization parameter.

As a consequence, working on each subdomain at one time we have

Theorem 6 The iterative process:

Given $\rho>0$ and $\lambda_{0} \in M$, find $u_{m} \in X$ for $m \geq 0$ via

$$
\begin{aligned}
\left(\nabla u_{m, 1}, \nabla v_{1}\right)_{\Omega_{1}}+\left(u_{m, 1}, v_{1}\right)_{\Omega_{1}} & =\left(f, v_{1}\right)_{\Omega_{1}}-\left(\lambda_{m}, v_{1}\right)_{1 / 2,00, \Gamma}, \quad \forall v_{1} \in X_{1}, \\
\left(\nabla u_{m, 2}, \nabla v_{2}\right)_{\Omega_{2}}+\left(u_{m, 2}, v_{2}\right)_{\Omega_{2}} & =\left(f, v_{2}\right)_{\Omega_{2}}+\left(\lambda_{m}, v_{2}\right)_{1 / 2,00, \Gamma}, \quad \forall v_{2} \in X_{2}, \\
\text { and update } & \\
\lambda_{m+1} & =\lambda_{m}+\rho\left(u_{m, 1}-u_{m, 2}\right) \quad \text { on } \Gamma
\end{aligned}
$$

is a non overlapping domain decomposition method geometrically convergent with a ratio of convergence independent of the mesh size. 
The drawback that this method presents is how to fix the optimal parameter $\rho>0$ but an estimate for a coarse grid will be enough. In the numerical experiments that we present the value of $\rho$ has been tuned easily by hand thanks to the great speed of convergence that the method exhibits.

For a method that has no need of fixing any parameter we show next the application of the Conjugate Gradient Method which is at the core of FETI methods (recall that $(\cdot, \cdot)_{1 / 2,00, \Gamma}$ is the scalar product on $M$ ):

Take $r_{0}=d_{0}=B R F-\left(B R B^{\star}\right) \lambda_{0}$, for $m \geq 0$ set $p_{m}:=\left(B R B^{\star}\right) d_{m}$ and repeat

$$
\begin{aligned}
\alpha_{m} & =\frac{\left(d_{m}, r_{m}\right)_{1 / 2,00, \Gamma}}{\left(d_{m}, p_{m}\right)_{1 / 2,00, \Gamma}} & & \\
\lambda_{m+1} & =\lambda_{m}+\alpha_{m} d_{m}, & & \text { on } \Gamma \\
r_{m+1} & =r_{m}-\alpha_{m} p_{m}, & & \text { on } \Gamma \\
\beta_{m} & =\frac{\left(p_{m}, r_{m+1}\right)_{1 / 2,00, \Gamma}}{\left(p_{m}, d_{m}\right)_{1 / 2,00, \Gamma}}, & & \\
d_{m+1} & =r_{m+1}-\beta_{m} d_{m}, & & \text { on } \Gamma .
\end{aligned}
$$

As before, using (28) the computation of the residual $r_{0}$ is made via the computation of $u_{0}$

$$
u_{0}=R F-\left(R B^{\star}\right) \lambda_{0} \Rightarrow r_{0}=B u_{0}
$$

and for the computation of $p_{m}:=\left(B R B^{\star}\right) d_{m}$ we set $p_{m}=B w_{m}$ where $w_{m}$ solve the auxiliar problem:

$$
R^{-1} w_{m}=B^{\star} d_{m} \quad \text { on } X^{\star}
$$

As before, the resolution of (43) is made on $\Omega_{1}$ and $\Omega_{2}$ independently. Therefore, this is also an iterative process that can be seen as a non overlapping domain decomposition method. Following standard convergence results, see for instance Quarteroni-Sacco-Saleri [21], we have geometric convergence in a finite number of steps (under exact arithmetic) for this iterative process. Suppose that $N$ is the size of the matrix $B R B^{\star}$, which amounts to say that $N$ is the number of degrees of freedom on the interfaces, then

Theorem 7 The method (37)-(41) converges geometrically in at most $N$ steps (under exact arithmetic). For any $m<N$ the error $e_{m}=\lambda_{m}-\lambda$ 
is orthogonal to the direction $d_{j}$ for $j=0,1,2, \ldots, m-1$ and we have the estimate

$$
\left\|\lambda_{m}-\lambda\right\|_{1 / 2,00, \Gamma} \leq 2 \sqrt{\kappa} \frac{c^{m}}{1+c^{2 m}}\left\|\lambda_{0}-\lambda\right\|_{1 / 2,00, \Gamma}
$$

where $c=(\sqrt{\kappa}-1) /(\sqrt{\kappa}+1)<1$ and $\kappa=\alpha^{2} / \beta^{2}$ is the spectral condition number of $B R B^{\star}$ that is independent of the discretization parameter.

\subsection{Case of floating subdomains}

A subdomain $\Omega_{r} \subset \Omega$ is called a floating subdomain when it does not touch $\partial \Omega$. As we have considered the bilinear form

$$
a(u, v)=\sum_{r=1}^{R}\left(u_{r}, v_{r}\right)_{1, \Omega_{r}}=\sum_{r=1}^{R} \int_{\Omega_{r}}\left\{\nabla u_{r} \cdot \nabla v_{r}+u_{r} v_{r}\right\} d x
$$

the ellipticity of the form $a(\cdot, \cdot)$ on $X$ is clear even when floating subdomains are present. When we consider the Laplace operator the bilinear form is

$$
\tilde{a}(u, v)=\sum_{r=1}^{R} \int_{\Omega_{r}} \nabla u_{r} \cdot \nabla v_{r} d x
$$

and the process above described could be called into question when solving the local, on each floating subdomain, problem because of the lack of ellipticity. But our process is still correct:

Suppose that $\Omega_{r} \subset \Omega$ is a floating subdomain and take $v \in X$ such that $v_{j}=v_{\left.\right|_{\Omega_{j}}}=0$ for $j \neq r$, then, as it is well known,

$$
\int_{\Omega_{r}} \nabla v_{r} \cdot \nabla v_{r} d x \Rightarrow v_{r}=\text { constant on } \Omega_{r}
$$

but the condition on the jumps $[v]_{\Gamma_{i}} \in H_{00}^{1 / 2}\left(\Gamma_{i}\right)$ for all $\forall \Gamma_{i} \in \mathcal{E}_{0}$ holds. Then, $v_{r}$ must equal the value zero on the cross points or corners on $\partial \Omega_{r}$ and, therefore, we obtain $v_{r}=0$ on $\Omega_{r}$. As a consequence, the ellipticity on floating subdomains is also obtained in this case. 


\section{$5 \quad$ Numerical experiments}

Several geometric configurations are considered: the unit box split into two subdomains, an inverted L-shape domain split in three subdomains, a square box split into four subdomains with an internal cross point and the same square box with a floating subdomain, diamond-like shaped with four corners. Our benchmark is

$$
\begin{array}{rll}
-\Delta u=f & \text { in } \Omega, \\
u=0 & \text { on } \partial \Omega .
\end{array}
$$

We take as initial Lagrange multiplier for our iteration process $\lambda_{0}^{i}=0$ on each interface $\Gamma_{i}$ between subdomains and stop iterating when the relative error between consecutive multipliers is small enough, for instance

$$
E_{r e l}\left(\lambda_{h}, m+1\right)=\frac{\sum_{i}\left\|\lambda_{m+1, h}^{i}-\lambda_{m, h}^{i}\right\|_{1 / 2,00, \Gamma_{i}}}{\sum_{i}\left\|\lambda_{m+1, h}^{i}\right\|_{1 / 2,00, \Gamma_{i}}} \leq 10^{-5}
$$

The ratio of geometric decay for the error $\sum_{i}\left\|\lambda_{m+1, h}-\lambda_{h}\right\|_{1 / 2,00, \Gamma_{i}}$ can also be estimated for $m$ large enough via

$$
r\left(\lambda_{h}\right) \approx \frac{E_{r e l}\left(\lambda_{h}, m+1\right)}{E_{r e l}\left(\lambda_{h}, m\right)}
$$

Then, several error estimates can be computed ( $R$ is the number of subdomains), for instance,

$$
\begin{aligned}
e u(h) & =\frac{\left(\int_{\Omega}\left|\nabla\left(u-u_{h}\right)\right|^{2} d x\right)^{1 / 2}}{\|u\|_{0, \Omega}} \\
\operatorname{eum}(h, m) & =\frac{\left(\sum_{i=1}^{R} \int_{\Omega_{i}}\left|\nabla\left(u-u_{i, h}^{m}\right)\right|^{2} d x\right)^{1 / 2}}{\|u\|_{0, \Omega}} \\
\operatorname{euhm}(h, m) & =\frac{\left(\sum_{i=1}^{R} \int_{\Omega_{i}}\left|\nabla\left(u_{h}-u_{i, h}^{m}\right)\right|^{2} d x\right)^{1 / 2}}{\left\|u_{h}\right\|_{0, \Omega}}
\end{aligned}
$$

where $u$ is the true solution, when available, $u_{h}$ is the Galerkin approximation computed on the global domain and $u_{i, h}^{m}$ is the approximation obtained on each step of the iteration process on each $\Omega_{i}$. The computations are performed with $\mathbb{P}_{1}$ finite elements on a family of triangulations of the whole computational domain such that the interfaces are formed by edges of triangles and their restrictions to each of the subdomains. The triangulations are uniform in all tests except the last one. 


\subsection{Two subdomains}

In $\Omega=(0,1) \times(0,1)$ we consider the exact solution of $(44)-(45)$ given by

$$
u(x, y)=\sin (2 \pi x) \cos (2 \pi y)-\sin (2 \pi x) .
$$

We take the interface $\Gamma \equiv\{y=0.25\}$, then $\Omega_{1}=(0,1) \times(0,0.25)$ and $\Omega_{2}=(0,1) \times(0.25,1)$. For Uzawa's Method we found by performing few several tests that $\rho \approx 0.12$ seems to be the closest value to the optimal one. Several results are shown in Table 1 . On the other hand, Table 2 shows the convergence results for the Conjugate Gradient method

\begin{tabular}{|c||c|c|c|c||}
\hline $1 / h$ & 8 & 16 & 32 & 64 \\
\hline$m=\#$ iterations & 10 & 12 & 13 & 14 \\
\hline \hline$\rho_{\text {opt }}$ & 0.15 & 0.13 & 0.12 & 0.12 \\
\hline$\left\|\lambda_{h}\right\|_{1 / 2,00, \Gamma}$ & $0.8319 \ldots$ & $0.8411 \ldots$ & $0.8438 \ldots$ & $0.8460 \ldots$ \\
\hline$r\left(\lambda_{h}\right)$ & $0.25 \ldots$ & $0.33 \ldots$ & $0.40 \ldots$ & $0.40 \ldots$ \\
\hline$e u(h)$ & $0.667 \ldots$ & $0.186 \ldots$ & $0.04788 \ldots$ & $0.0120 \ldots$ \\
\hline eum $(h, m)$ & $0.667 \ldots$ & $0.186 \ldots$ & $0.04788 \ldots$ & $0.0120 \ldots$ \\
\hline euhm $(h, m)$ & $6.09 \ldots \mathrm{e}-6$ & $6.26 \ldots \mathrm{e}-6$ & $9.6 \ldots \mathrm{e}-6$ & $5.47 \ldots \mathrm{e}-6$ \\
\hline \hline
\end{tabular}

Table 1: Two subdomains with Uzawa's Method: Number of iterations, values of $\rho_{\text {opt }},\left\|\lambda_{h}\right\|_{1 / 2,00, \Gamma}, r\left(\lambda_{h}\right)$ and several other error estimates for different values of $h$.

\begin{tabular}{|c||c|c|c|c||}
\hline $1 / h$ & 8 & 16 & 32 & 64 \\
\hline$m=$ \#iterations & 6 & 8 & 9 & 9 \\
\hline \hline$\left\|\lambda_{h}\right\|_{1 / 2,00, \Gamma}$ & $0.8319 \ldots$ & $0.8411 \ldots$ & $0.8438 \ldots$ & $0.8460 \ldots$ \\
\hline$r\left(\lambda_{h}\right)$ & $0.11 \ldots$ & $0.15 \ldots$ & $0.213 \ldots$ & $0.221 \ldots$ \\
\hline$e u(h)$ & $0.667 \ldots$ & $0.186 \ldots$ & $0.04788 \ldots$ & $0.0120 \ldots$ \\
\hline $\operatorname{eum}(h, m)$ & $0.667 \ldots$ & $0.186 \ldots$ & $0.04788 \ldots$ & $0.0120 \ldots$ \\
\hline euhm $(h, m)$ & $8.6 \ldots \mathrm{e}-7$ & $3.6 \ldots \mathrm{e}-7$ & $2.3 \ldots \mathrm{e}-6$ & $3.2 \ldots \mathrm{e}-6$ \\
\hline \hline
\end{tabular}

Table 2: Two subdomains with Conjugate Gradient Method: Number of iterations, values of $\left\|\lambda_{h}\right\|_{1 / 2,00, \Gamma}, r\left(\lambda_{h}\right)$ and several other error estimates for different values of $h$.

For both iterative methods Figure 1 shows the decay of the error as a function of the number of iterations, measured by (46) for the Lagrange multiplier and Figure 2 shows the error, given by (50) (base-10 logarithmic scale is used on 
the y-axis), also as a function of the number of iterations. Figure 3 shows the decay ratio given by (47) for the convergence on the Lagrange multiplier.

As we see, Conjugate Gradient method performs better and, therefore, our following numerical tests will be performed with this iterative method.

\subsection{Non convex domain with three subdomains}

We consider an inverted L-shaped domain $\Omega=\{(-1,1) \times(-1,1)\} \backslash\{(-1,0) \times$ $(-1,0)\}$ and decompose it into three squares given by $\Omega_{1}=(-1,0) \times(0,1)$, $\Omega_{2}=(0,1) \times(0,1)$ and $\Omega_{3}=(0,1) \times(-1,0)$ so that our interfaces are $\Gamma_{1}=\{0\} \times(0,1)$ and $\Gamma_{2}=(0,1) \times\{0\}$. We solve (44)-(45) with right hand side given by

$$
f(x, y)=\left\{\begin{array}{rrl}
10 & \text { when } x^{2}+y^{2} \leq 0.25 & \text { and } y<0 \\
-10 & \text { when } x^{2}+y^{2} \leq 0.25 & \text { and } y<0, x>0 \\
0 & \text { elsewhere }
\end{array}\right.
$$

The results are shown in Table 3 .

\begin{tabular}{|c||c|c|c|c||}
\hline $1 / h$ & 4 & 8 & 16 & 32 \\
\hline$m=\#$ iterations & 6 & 8 & 10 & 10 \\
\hline \hline$\left\|\lambda_{h}^{1}\right\|_{1 / 2,00, \Gamma_{1}}$ & $0.0469 \ldots$ & $0.1085 \ldots$ & $0.1463 \ldots$ & $0.1680 \ldots$ \\
\hline$\left\|\lambda_{h}^{2}\right\|_{1 / 2,00, \Gamma_{2}}$ & $0.1402 \ldots$ & $0.2448 \ldots$ & $0.3137 \ldots$ & $0.3550 \ldots$ \\
\hline$r\left(\lambda_{h}\right)$ & $0.01 \ldots$ & $0.13 \ldots$ & $0.15 \ldots$ & $0.21 \ldots$ \\
\hline euhm $(h, m)$ & 0 & $6.15 \ldots \mathrm{e}-7$ & $3.9 \ldots \mathrm{e}-7$ & $1.5 \ldots \mathrm{e}-6$ \\
\hline \hline
\end{tabular}

Table 3: Inverted L-shape domain split into three subdomains: Number of iterations, values of $\left\|\lambda_{h}^{i}\right\|_{1 / 2,00, \Gamma_{i}}, r\left(\lambda_{h}\right)$ and $\operatorname{euhm}(h, m)$ for different $h$. Conjugate Gradient Method is used.

For this L-shape test, Figure 4 shows the decay of the error, given by (46), for the Lagrange multiplier and the decay of the error for the solution, given by (50) (base-10 logarithmic scale on the y-axis). Also the decay ratio given by (47) for the convergence on the Lagrange multiplier is shown; all of them as a function of the number of iterations. Figure 5 shows the $\mathbb{P}_{1}$ Galerkin solution on the whole domain for $h=1 / 32$. Figure 6 the computed solution with domain decomposition, Figure 7 shows the iterate obtained for $\lambda_{0}^{i}=0$ and Figure 8 the second iterate on the process. 


\subsection{Four subdomains: case of an internal cross point}

We take now $\Omega=(-1,1)^{2}=\cup_{i=1}^{4} \Omega_{i}$ where $\Omega_{1}=(-1,0) \times(0,1), \Omega_{2}=$ $(0,1) \times(0,1), \Omega_{3}=(0,1) \times(-1,0)$ and $\Omega_{4}=(-1,0) \times(-1,0)$. Then the interfaces are $\Gamma_{1}=(-1,0) \times\{0\}, \Gamma_{2}=\{0\} \times(0,1), \Gamma_{3}=(0,1) \times\{0\}$ and $\Gamma_{4}=\{0\} \times(-1,0)$ and all meet on $P=(0,0)$. We solve (44)-(45) with right hand side given by

$$
f(x, y)=10^{3} \sin (6 x-7 y) \cos (10 x+4 y) .
$$

Again we use a uniform triangular mesh of mesh size $h$ of $\bar{\Omega}$, its restriction to each of the $\overline{\Omega_{i}}$ for $i=1,2,3,4$ and $\mathbb{P}_{1}$ finite elements on each computational domain.

In this case the symmetric matrix $R B^{\star}$ of the linear system to solve has a block independent structure, each block related to each one of the subdomains that meets on the cross point, and a single row that relates all of them. The resolution of the linear systems, with matrix $R B^{\star}$, that appear on each step of the conjugate gradient process is performed via a simple iteration process that can be performed on each subdomain separately. A detailed description will be revealing:

Denote by $\left\{\phi_{1}, \phi_{2}, \ldots, \phi_{n n t}\right\}$ the $\mathbb{P}_{1}$ basis hat functions on the triangulation of $\bar{\Omega}$, where nnt is the total number of nodes, and set $\phi_{p}$ the hat function associated to the cross point $P$. Then, denote by $\left\{\varphi_{j}^{i}\right\}$ for $j=1, \ldots, n t i$ the restrictions of these $\phi_{s}$ to each $\overline{\Omega_{i}}$, where $n t i$ is the total number of nodes on each $\overline{\Omega_{i}}$, and set $j=n t c i$ the index such that $\varphi_{n t c i}^{i}=\phi_{p_{\left.\right|_{\Omega_{i}}}}$. Denote by $X_{h}$, the internal approximation of $X$ with $\mathbb{P}_{1}$ finite elements, then for $u \in X_{h}$ the fact that $[u]_{\Gamma}=0$ on all interfaces implies that

$$
u=\sum_{r=1}^{4} \sum_{\substack{j=1 \\ j \neq n t c r}}^{n t r} \alpha_{j}^{r} \varphi_{j}^{r}+\alpha_{p} \phi_{p}, \quad u(P)=\alpha_{p} .
$$

Then, $\operatorname{dim} X_{h}=\sum_{r=1}^{4} n t r-3$ and the set of basis functions for $X_{h}$ is

$$
\left\{\left\{\varphi_{j}^{r}\right\}_{\{r=1, \ldots, 4, j=1, \ldots, n t r, j \neq n t c r\}}, \phi_{p}\right\}
$$

As a consequence, the search of $u \in X_{h}$ such that for all $v \in X_{h}$ satisfies

$$
a(u, v)=l(v)
$$


leads to a linear symmetric system with a block matrix structure like

$$
\left(\begin{array}{ccccc}
A^{1} & 0 & 0 & 0 & c^{1} \\
0 & A^{2} & 0 & 0 & c^{2} \\
0 & 0 & A^{3} & 0 & c^{3} \\
0 & 0 & 0 & A^{4} & c^{4} \\
c^{1, t} & c^{2, t} & c^{3, t} & c^{3, t} & c_{p}
\end{array}\right)\left(\begin{array}{c}
\alpha^{1} \\
\alpha^{2} \\
\alpha^{3} \\
\alpha^{4} \\
\alpha_{p}
\end{array}\right)=\left(\begin{array}{l}
l^{1} \\
l^{2} \\
l^{3} \\
l^{4} \\
l_{p}
\end{array}\right)
$$

where for $r=1,2,3,4$ and $i, j=1, \ldots, n t r, i, j \neq n t c r$ we have

$$
\begin{aligned}
A^{r} & =\left(a_{i, j}^{r}\right)_{i, j}, \quad a_{i, j}^{r}=a\left(\varphi_{i}^{r}, \varphi_{j}^{r}\right), \\
c^{r} & =\left(c_{j}^{r}\right)_{j}, \quad c_{j}^{r}=a\left(\phi^{p}, \varphi_{j}^{r}\right), \quad c_{p}=a\left(\phi_{p}, \phi_{p}\right) \in \mathbb{R} \\
l^{r} & =\left(l_{j}^{r}\right)_{j}, \quad l_{j}^{r}=l\left(\varphi_{j}^{r}\right), \quad l_{p}=l\left(\phi_{p}\right) \in \mathbb{R} \\
\alpha^{r} & =\left(\alpha_{j}^{r}\right)_{j}, \quad \alpha_{p} \in \mathbb{R}
\end{aligned}
$$

( $c^{r, t}$ denotes transpose). Almost all entries on each $c^{r}$ are zero except for those nodes that are neighbours of the cross point $P$ on $\Omega_{r}$. In our uniform triangulation, we have only two or three nonzero entries on each $c^{r}$. Then, coupling is solved via a simple relaxation process that allows the computation separately on each subdomain as follows:

We set $\alpha_{m}^{r}=0, \alpha_{p, m}=0$ for $m=0$ and $r=1,2,3,4$ and solve for each $r$

$$
\left(\begin{array}{cc}
A^{r} & c^{r} \\
c^{r, t} & c_{p}
\end{array}\right)\left(\begin{array}{c}
\alpha_{m+1}^{r} \\
\alpha_{p, m+1}
\end{array}\right)=\left(\begin{array}{c}
l^{r} \\
l_{p}
\end{array}\right)-\left(\begin{array}{c}
0 \\
\sum_{s<r} c^{s, t} \alpha_{m+1}^{s}+\sum_{s>r} c^{s, t} \alpha_{m}^{s}
\end{array}\right),
$$

observe that $s, r \in\{1,2,3,4\}$ with the usual convention that the sums are zero whenever the sets $\{s<r\}$ or $\{s>r\}$ are empty and that, as we mentioned before, the connectivity vectors $c^{s}$ have almost all entries zero. As a consequence, for each $m$ we update the value of $\alpha_{p, m+1}$ once for each subdomain. A fast convergence to the solution, fixed point, for this process has been obtained. The results are shown in Table 4 .

Figure 9 shows the decay of the error, given by (50), between the $\mathbb{P}_{1^{-}}$ Galerkin solution on the whole domain and the solution computed via domain decomposition, the decay of the error, given by (46), for the Lagrange multipliers (base-10 logarithmic scale on the y-axis) and the decay ratio given by (47), as a function of the number of iterations, for the convergence on the Lagrange multipliers for different values of $h$. Figure 10 shows the Galerkin solution computed for $h=1 / 32$, Figure 11 shows the initial domain decomposition iterate we depart from and Figure 12 shows the final domain decomposition solution for $h=1 / 32$. 


\begin{tabular}{|c||c|c|c|c||}
\hline $1 / h$ & 4 & 8 & 16 & 32 \\
\hline$m=\#$ iterations & 4 & 7 & 8 & 8 \\
\hline \hline$\left\|\lambda_{h}^{1}\right\|_{1 / 2,00, \Gamma_{1}}$ & $2.05 \ldots$ & $4.05 \ldots$ & $4.93 \ldots$ & $5.16 \ldots$ \\
\hline$\left\|\lambda_{h}^{2}\right\|_{1 / 2,00, \Gamma_{2}}$ & $1.66 \ldots$ & $3.12 \ldots$ & $3.65 \ldots$ & $3.80 \ldots$ \\
\hline$\left\|\lambda_{h}^{3}\right\|_{1 / 2,00, \Gamma_{3}}$ & $2.05 \ldots$ & $4.05 \ldots$ & $4.93 \ldots$ & $5.16 \ldots$ \\
\hline$\left\|\lambda_{h}^{4}\right\|_{1 / 2,00, \Gamma_{4}}$ & $1.66 \ldots$ & $3.12 \ldots$ & $3.65 \ldots$ & $3.80 \ldots$ \\
\hline$r\left(\lambda_{h}\right)$ & $0.09 \ldots$ & $0.12 \ldots$ & $0.20 \ldots$ & $0.21 \ldots$ \\
\hline euhm $(h, m)$ & $9.0 \ldots \mathrm{e}-15$ & $4.3 \ldots \mathrm{e}-15$ & $1.32 \ldots \mathrm{e}-5$ & $3.1 \ldots \mathrm{e}-5$ \\
\hline \hline
\end{tabular}

Table 4: Four subdomains with a cross point: Number of iterations, values of $\left\|\lambda_{h}^{i}\right\|_{1 / 2,00, \Gamma_{i}}, r\left(\lambda_{h}\right)$ and $\operatorname{euhm}(h, m)$ for different values of $h$.

\subsection{Case of a floating subdomain: four crosspoints}

As before we take $\Omega=(-1,1)^{2}$ and consider now $\Omega_{i}$ the convex hull generated by the points $P_{1}=(-0.5,0), P_{2}=(0,0.5), P_{3}=(0.5,0)$ and $P_{4}=(0,-0.5)$ and $\Omega_{e}=\Omega \backslash \overline{\Omega_{i}}$. Then the interfaces are the sides of this diamond shaped domain. We also solve (44)-(45) with the same right hand side as in the previous test but now on triangulations are no longer uniform. We consider triangulations of $\bar{\Omega}$ conforming with the interfaces and their restrictions to $\overline{\Omega_{i}}$ and $\overline{\Omega_{e}}$. On each computational domain $\mathbb{P}_{1}$ finite elements are used and the $\mathbb{P}_{1}$-Galerkin solution on $\Omega$ has already been shown on Figure 10 .

We proceed as in the previous example because the symmetric matrix $R B^{\star}$ has a two-block independent structure coupled by four sparse rows, one for each point $P_{i}$. The results are shown in Table 5 .

\begin{tabular}{|c||c|c|c||}
\hline nodes on $\overline{\Omega_{i}}$ & 128 & 393 & 687 \\
\hline nodes on $\overline{\Omega_{e}}$ & 894 & 2627 & 4621 \\
\hline$m=\#$ iterations & 9 & 10 & 10 \\
\hline \hline$\left\|\lambda_{h}^{1}\right\|_{1 / 2,00, \Gamma_{1}}$ & $4.30 \ldots$ & $4.41 \ldots$ & $4.43 \ldots$ \\
\hline$\left\|\lambda_{h}^{2}\right\|_{1 / 2,00, \Gamma_{2}}$ & $6.62 \ldots$ & $6.66 \ldots$ & $6.69 \ldots$ \\
\hline$\left\|\lambda_{h}^{3}\right\|_{1 / 2,00, \Gamma_{3}}$ & $4.30 \ldots$ & $4.41 \ldots$ & $4.43 \ldots$ \\
\hline$\left\|\lambda_{h}^{4}\right\|_{1 / 2,00, \Gamma_{4}}$ & $6.44 \ldots$ & $6.63 \ldots$ & $6.70 \ldots$ \\
\hline$r\left(\lambda_{h}\right)$ & $0.26 \ldots$ & $0.16 \ldots$ & $0.26 .$. \\
\hline$e u h m(h)$ & $5.17 \ldots \mathrm{e}-07$ & $8.5 \ldots \mathrm{e}-07$ & $4.39 \ldots \mathrm{e}-07$ \\
\hline \hline
\end{tabular}

Table 5: Floating subdomain with four cross points: Number of iterations, values of $\left\|\lambda_{h}^{i}\right\|_{1 / 2,00, \Gamma_{i}}, r\left(\lambda_{h}\right)$ and $\operatorname{euhm}(h, m)$ for different triangulations. 
Figure 13 shows the decay of the error, given by (50), between the $\mathbb{P}_{1}$-Galerkin solution on the whole domain and the solution computed via domain decomposition, the decay of the error, given by (46), for the Lagrange multipliers (base-10 logarithmic scale on the y-axis) and the decay ratio given by (47), as a function of the number of iterations, for the convergence on the Lagrange multipliers for different values of $h$. Conjugate Gradient Method is used. Figure 14 shows two meshes and Figure 15 the computed solution on $\Omega_{i}$ and on $\Omega_{e}$ for the finest mesh of 687 and 4621 nodes respectively. 


\section{Conclusions}

As far as we know, the approach to FETI methods has been made mostly from a linear algebra point of view. Some new aspects have been given in this paper: Cross points and floating subdomains are handled quite naturally, we only care about the interior of the interfaces and, thanks to the finite element extension results, the direct computation of the $H_{00}^{1 / 2}(\Gamma)$ scalar product yields an iteration matrix with a condition number independent of the mesh size. Therefore, we obtain a mesh independent ratio of convergence for the iterative methods. Moreover, no preconditioning is needed. This result improves the standard asymptotic bound for this condition number given by $C(1+$ $\log (H / h))^{2}$, where $H$ and $h$ are the characteristic subdomain size and element size respectively, shown by Mandel-Tezaur in [19].

In the two dimensional setting the computation of the $H^{1 / 2}$ scalar product for the $\mathbb{P}_{1}$ discrete basis functions on the interfaces is not expensive and it is performed once as long as the mesh does not change on these interfaces. Numerical tests have been presented where the convergence ratio is mesh independent according with the theoretical results. Three dimensional configurations will be studied in future works.

Acknowledgments: Research partially funded by Spanish government MEC Research Project MTM2006-02175. 

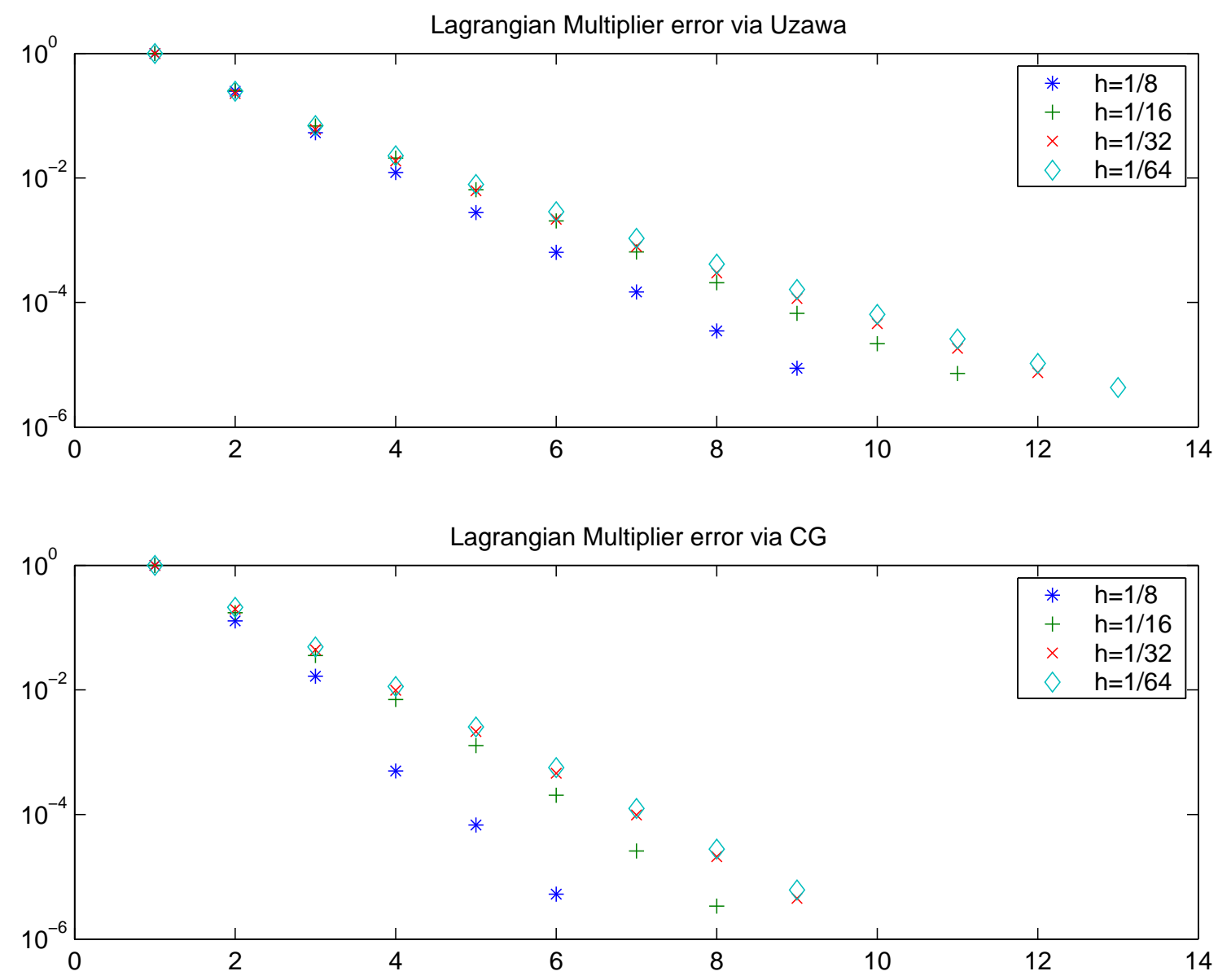

Figure 1: Unit box with two subdomains: Decay of the error, as a function of the number of iterations, given by (46), for the Lagrange multiplier using Uzawa's Method and Conjugate Gradient Method (CG) (base-10 logarithmic scale on the y-axis). 

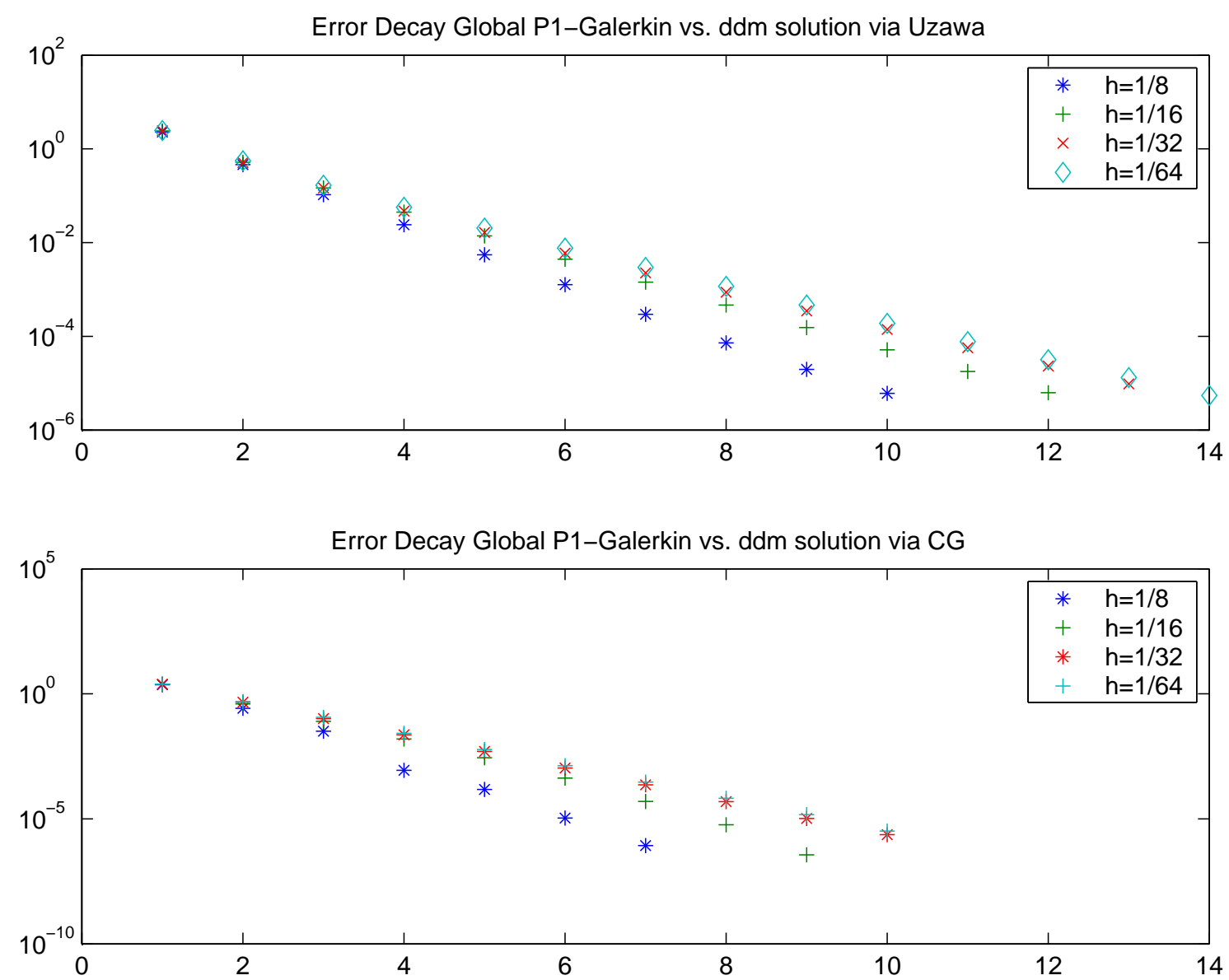

Figure 2: Unit box with two subdomains: Decay of the error, as a function of the number of iterations, given by (50), for the $\mathbb{P}_{1}$-Galerkin solution on the whole domain and the solution computed via domain decomposition, using Uzawa's Method and Conjugate Gradient Method (CG) (base-10 logarithmic scale on the $y$-axis). 

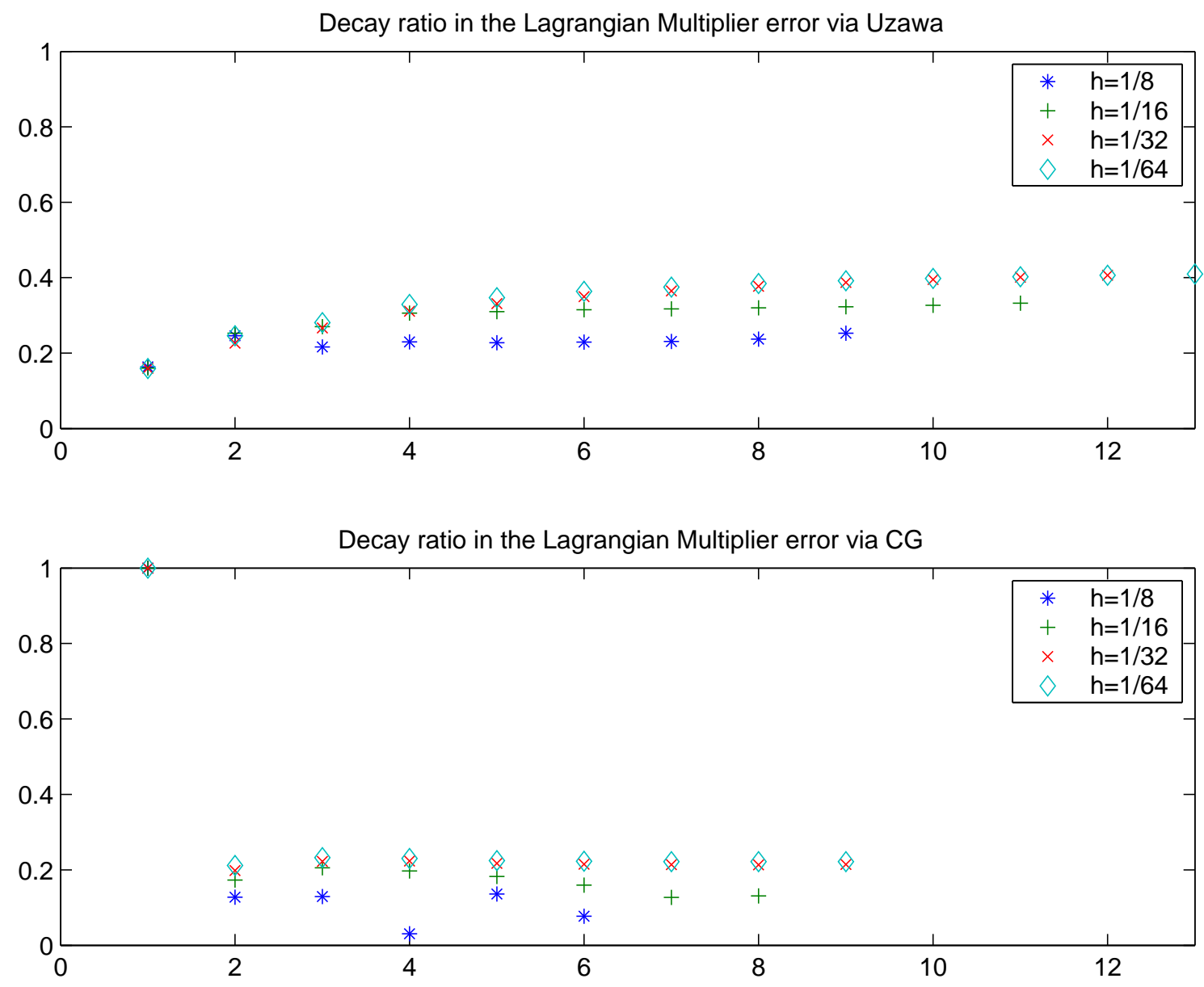

Figure 3: Unit box with two subdomains: Decay ratio, as a function of the number of iterations, given by (47) for the convergence on the Lagrange multiplier using Uzawa's Method and Conjugate Gradient Method (CG). 

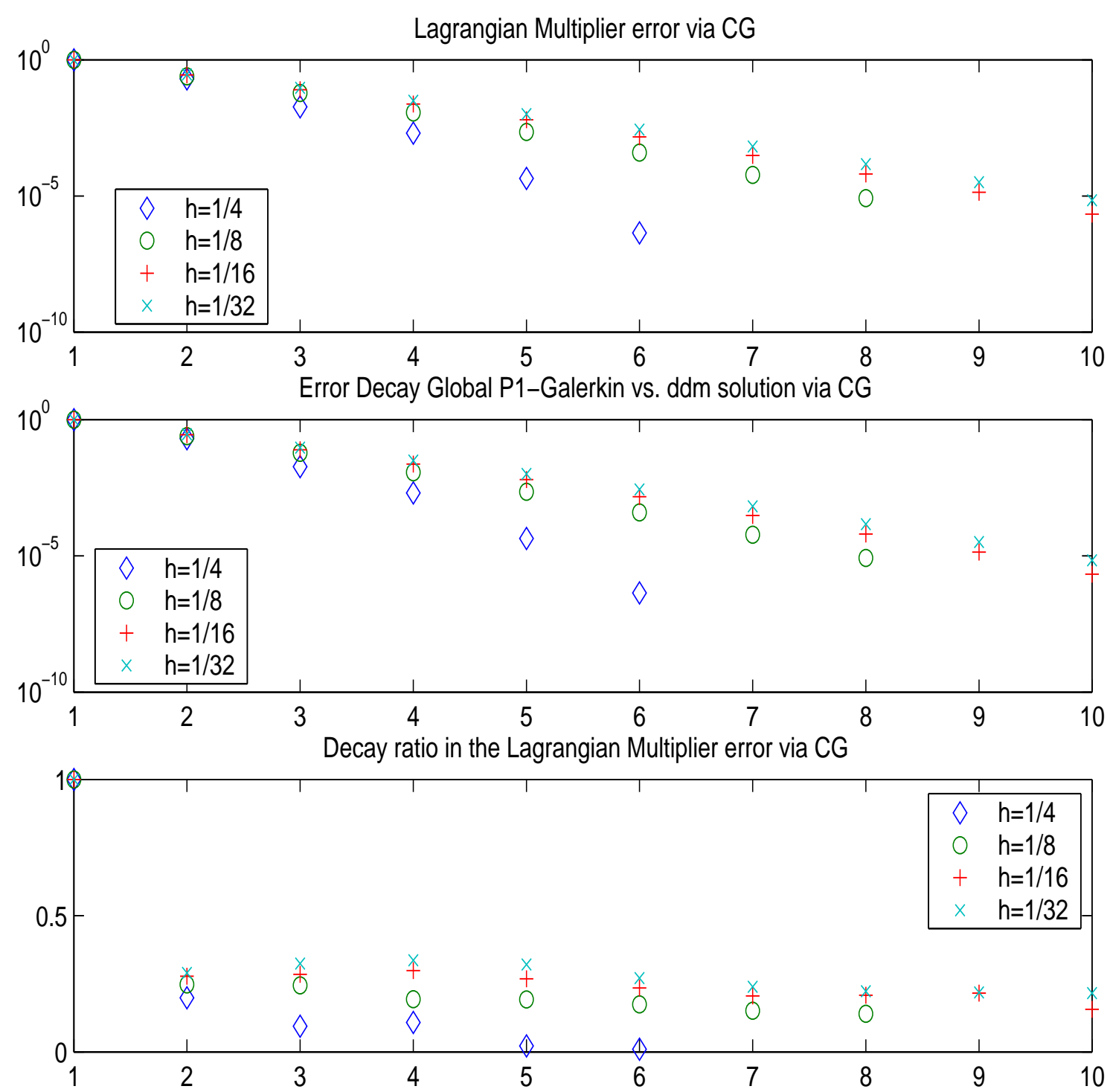

Figure 4: L-shape with three subdomains: Decay of the error, as a function of the number of iterations, given by (50), for the $\mathbb{P}_{1}$-Galerkin solution on the whole domain and the solution computed via domain decomposition; Decay of the error, given by (46), for the Lagrange multiplier (base-10 logarithmic scale on the y-axis); Decay ratio given by (47) for the convergence on the Lagrange multiplier. Conjugate Gradient Method is used. 


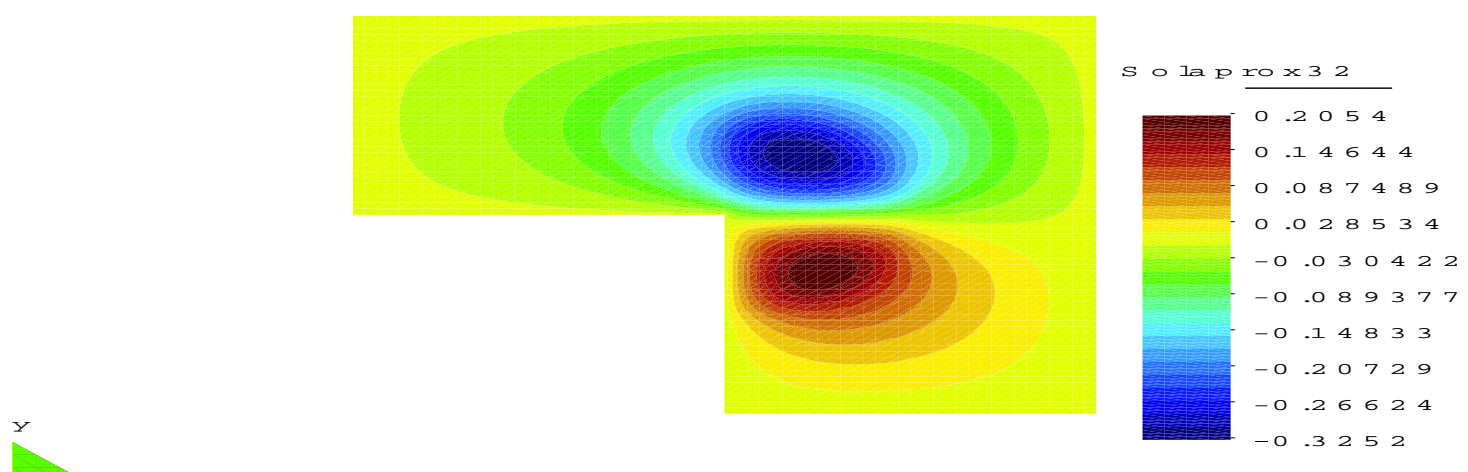

C on tour $\mathrm{F}$ ill of $\mathrm{s}$ olaprox 32 .

Figure 5: L-shape with three subdomains: $\mathbb{P}_{1}$ Galerkin solution on the whole domain with $h=1 / 32$.

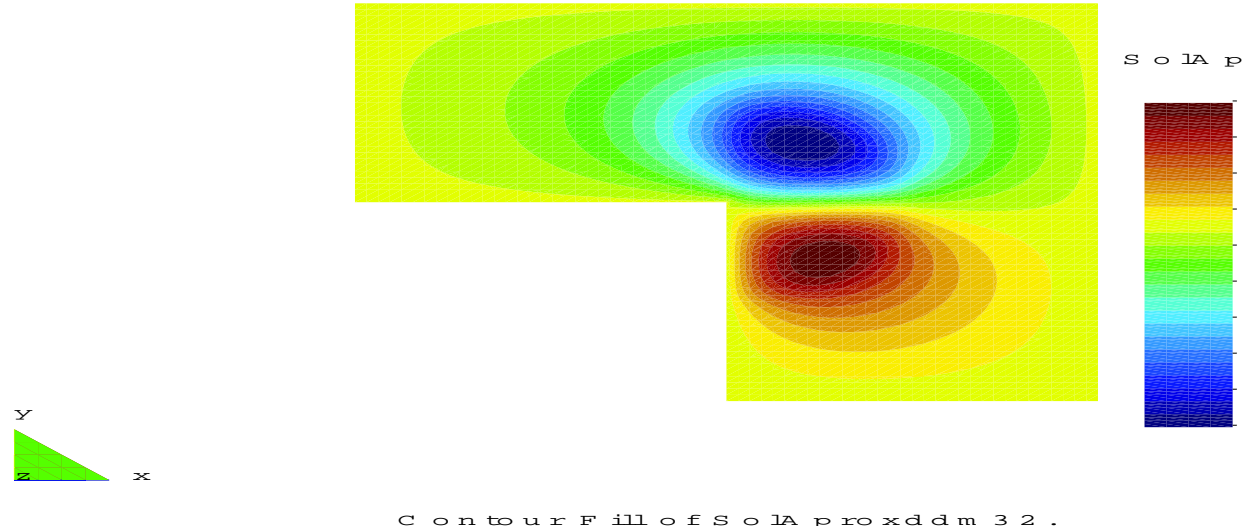

C On tour F ill of $S$ O IA p roxddm 32 .

Figure 6: L-shape with three subdomains: Domain decomposition solution computed with $h=1 / 32$. 


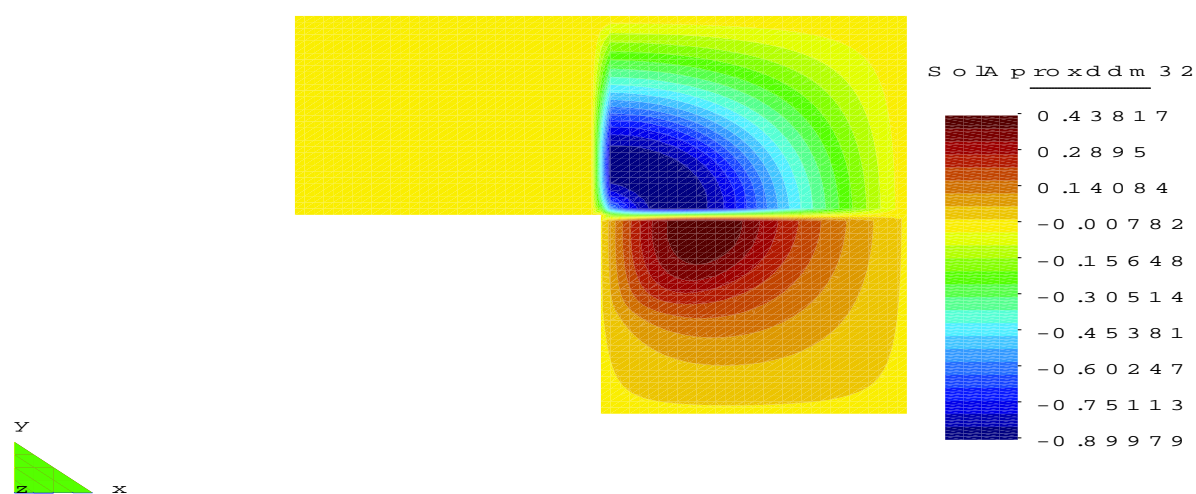

C On tourF ill OfS I I p roxddm 32 .

GiD

Figure 7: L-shape with three subdomains: Initial solution for the iteration process with $h=1 / 32$.

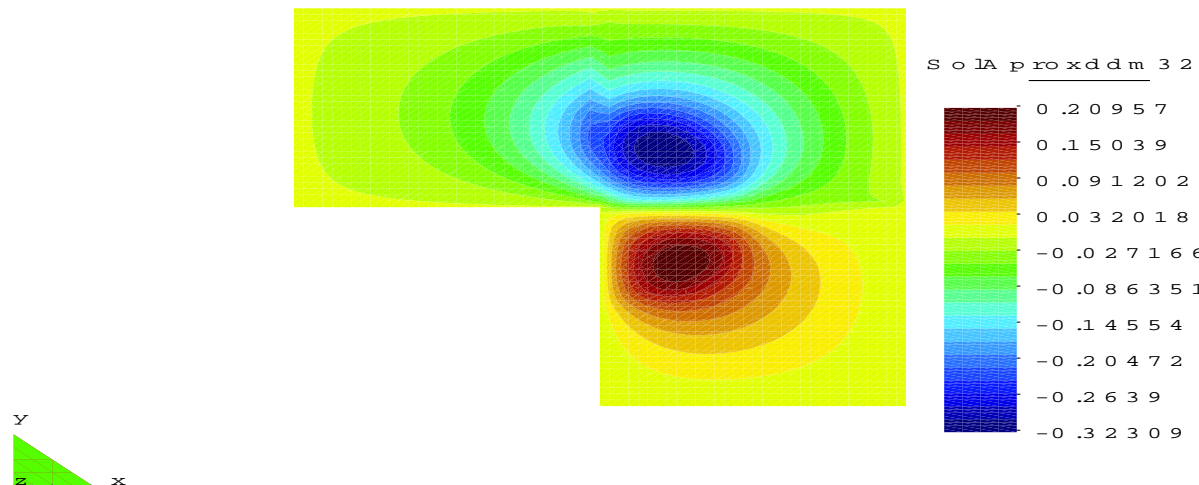

C On tourF ill Of S O IA proxddm 32

Figure 8: L-shape with three subdomains: Solution after two iterations for $h=1 / 32$. 

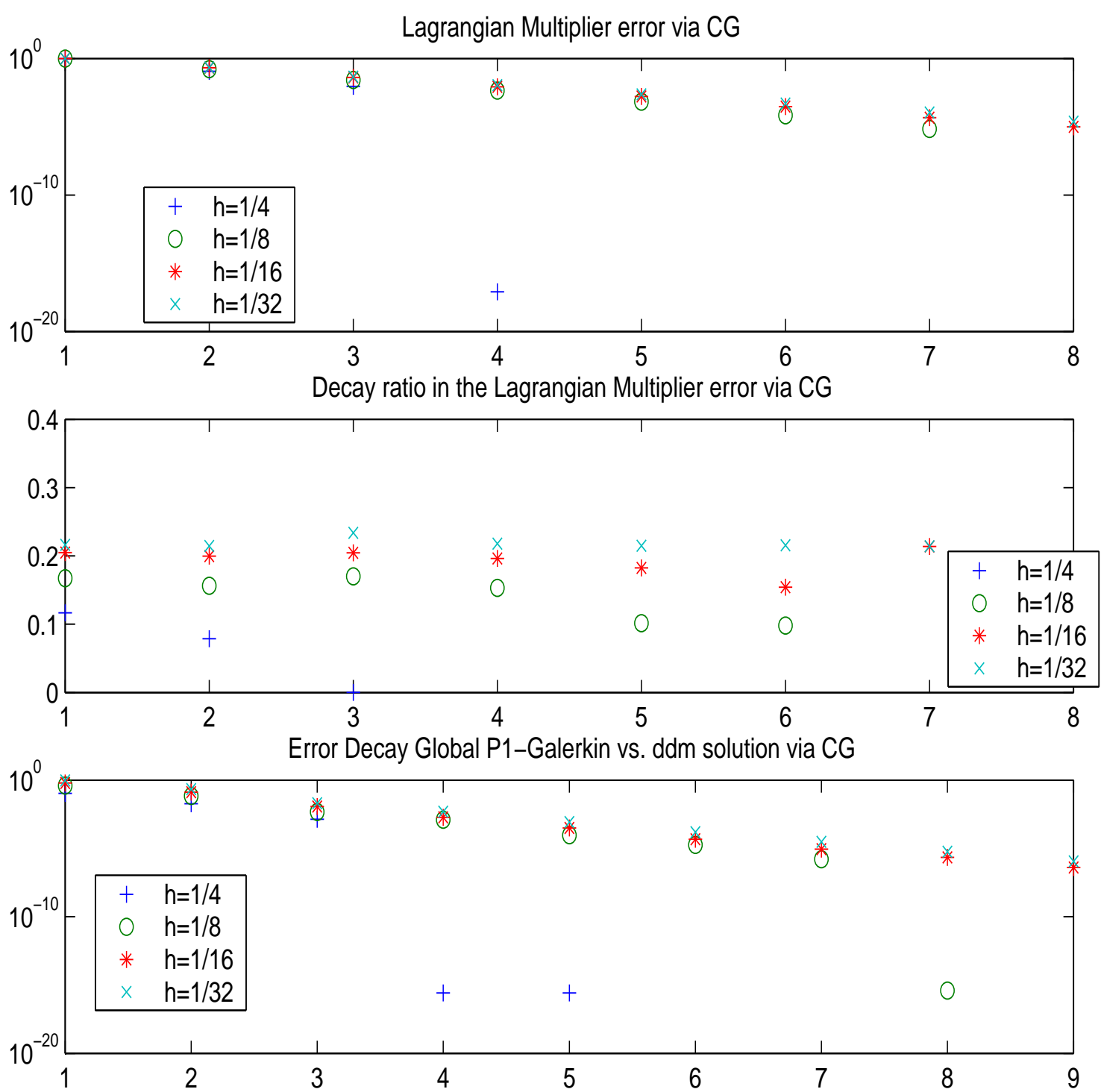

Figure 9: Square box with four subdomains: Decay of the error, as a function of the number of iterations, given by (50), between the $\mathbb{P}_{1}$-Galerkin solution on the whole domain and the solution computed via domain decomposition; Decay of the error, given by (46), for the Lagrange multiplier, (base-10 logarithmic scale on the $y$-axis); Decay ratio given by (47) for the convergence on the Lagrange multiplier. Conjugate Gradient Method is used. 


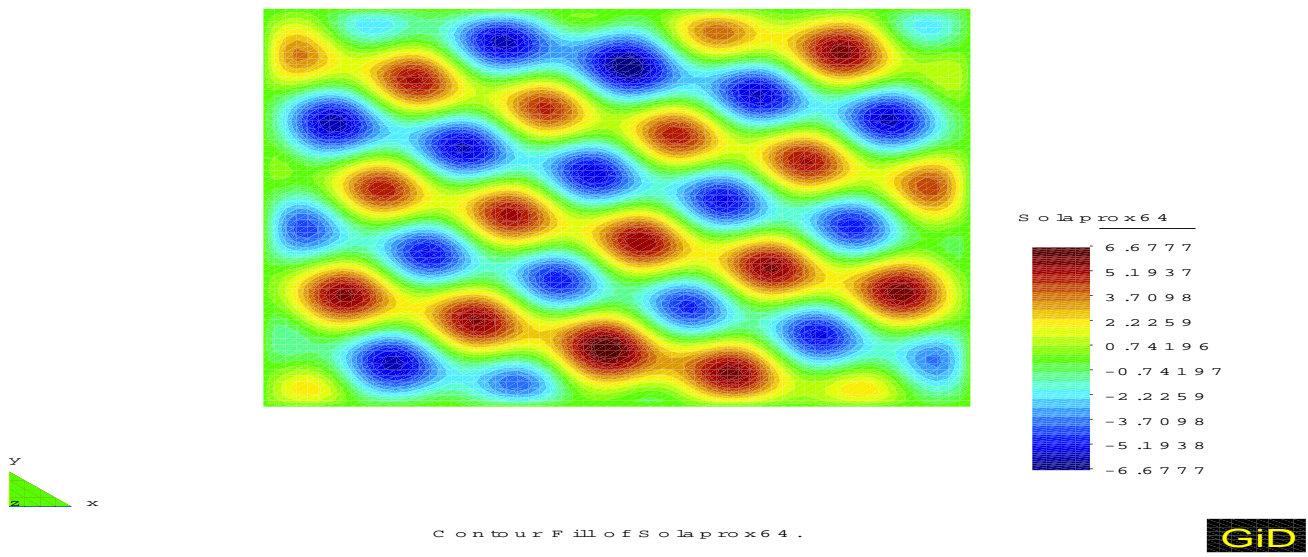

Figure 10: Square box with four subdomains: Galerkin solution computed for $h=1 / 32$.

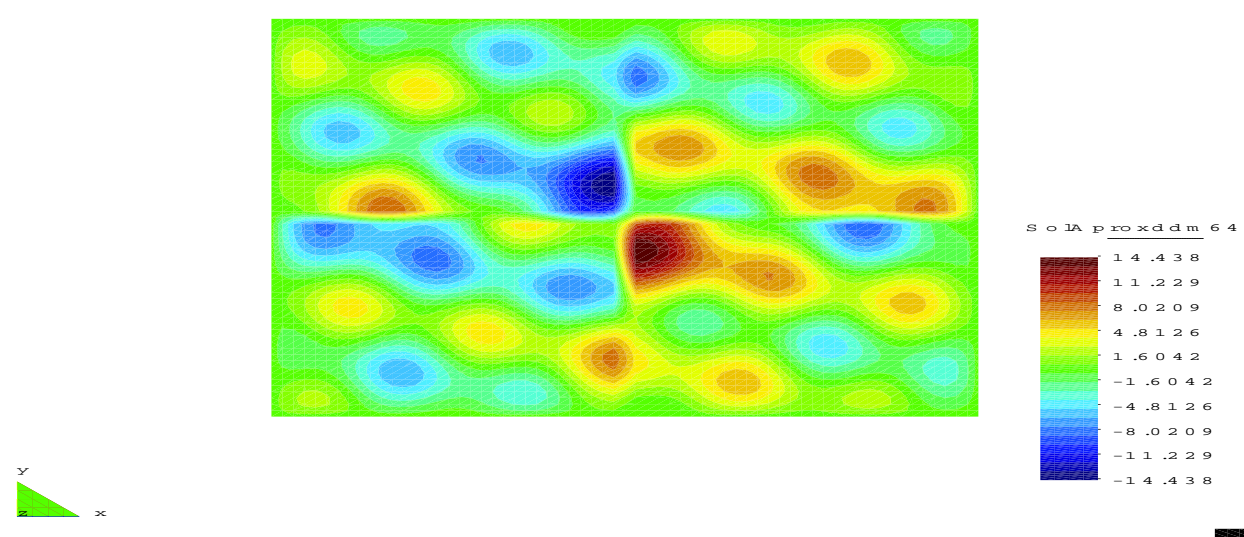

GiD

Figure 11: Square box with four subdomains: Initial iterate for $h=1 / 32$. 


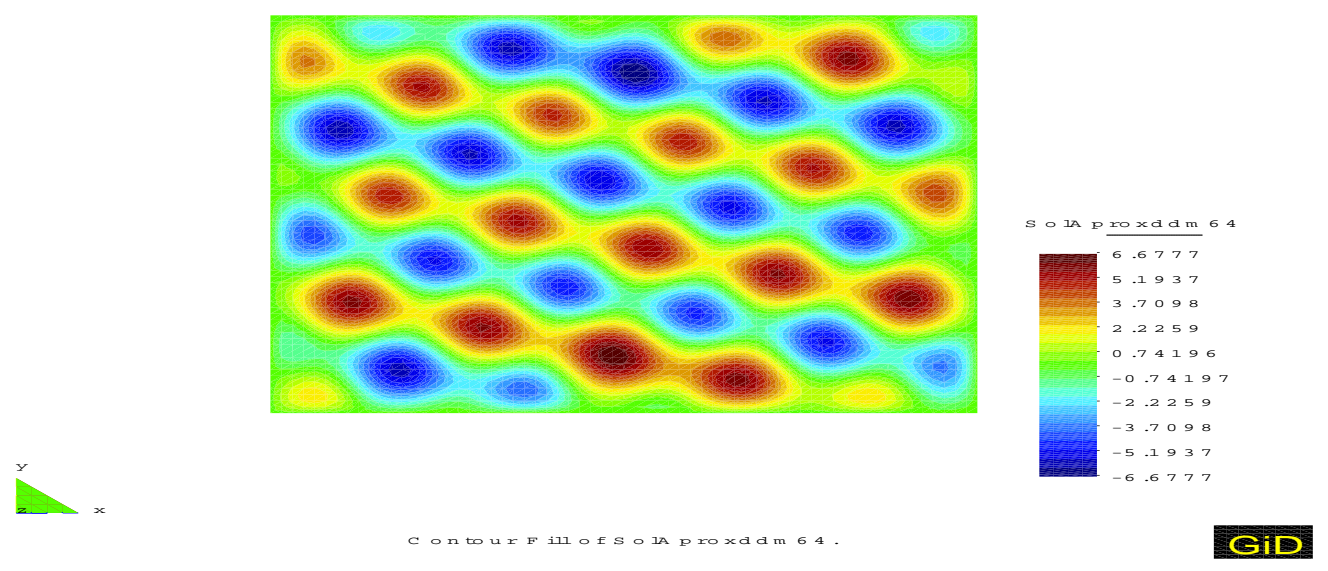

Figure 12: Square box with four subdomains: Domain decomposition solution computed with $h=1 / 32$. 

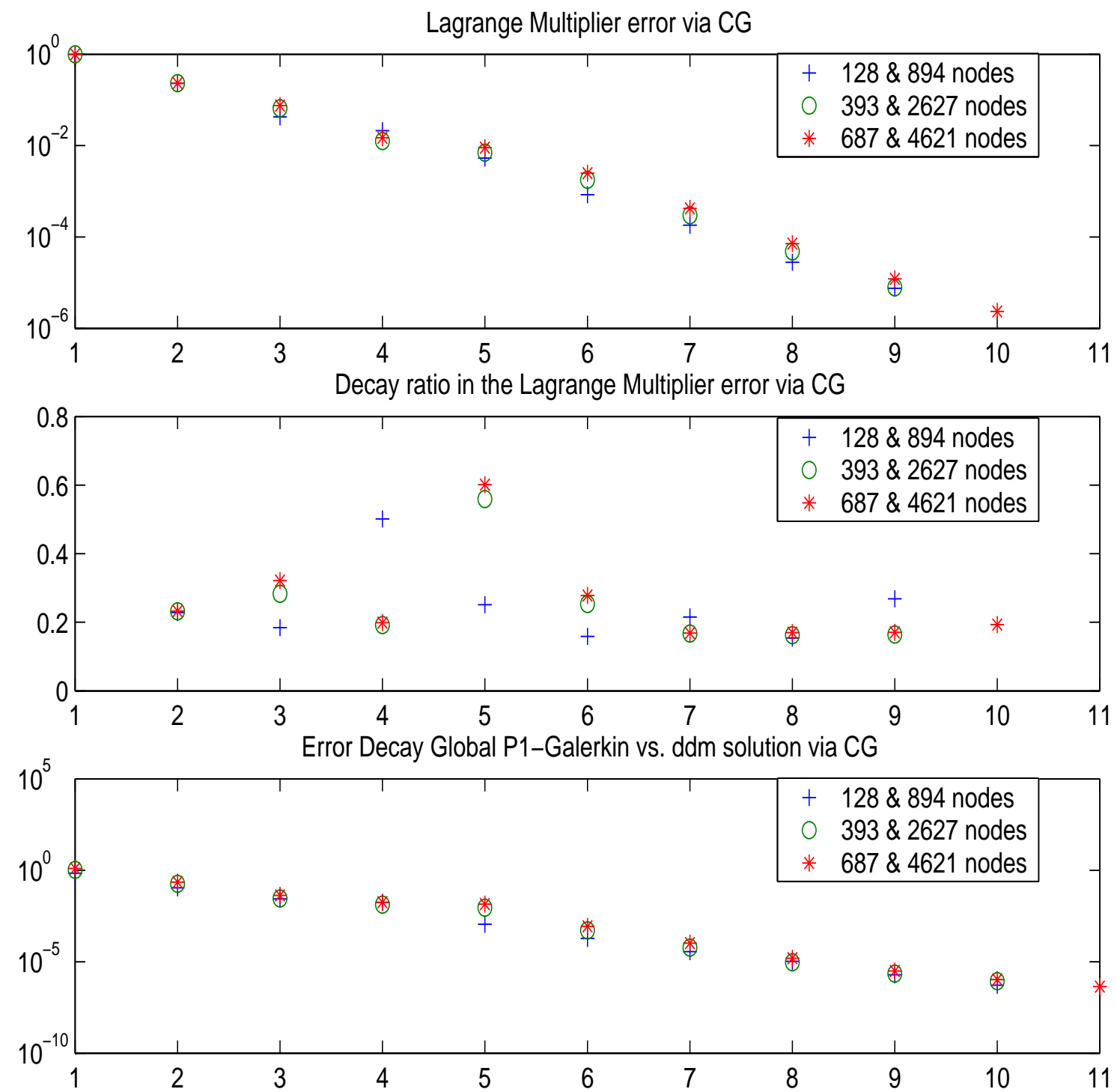

Figure 13: Square box with floating subdomain: Decay of the error, as a function of the number of iterations, given by (50), between the $\mathbb{P}_{1}$-Galerkin solution on the whole domain and the solution computed via domain decomposition; Decay of the error, given by (46), for the Lagrange multiplier, (base-10 logarithmic scale on the $y$-axis); Decay ratio given by (47) for the convergence on the Lagrange multiplier. Conjugate Gradient Method is used. 


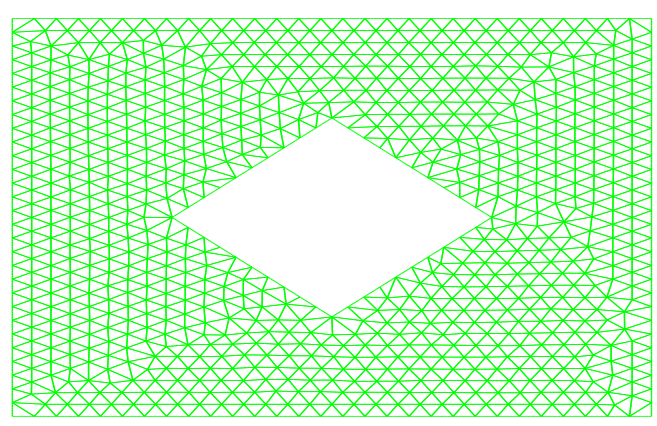

GiD

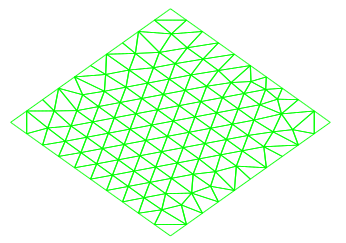

GiD

Figure 14: Square box with a floating subdomain: Triangulation on floating (214 triangles and 128 nodes) and external subdomain (1632 triangles and 894 nodes). 


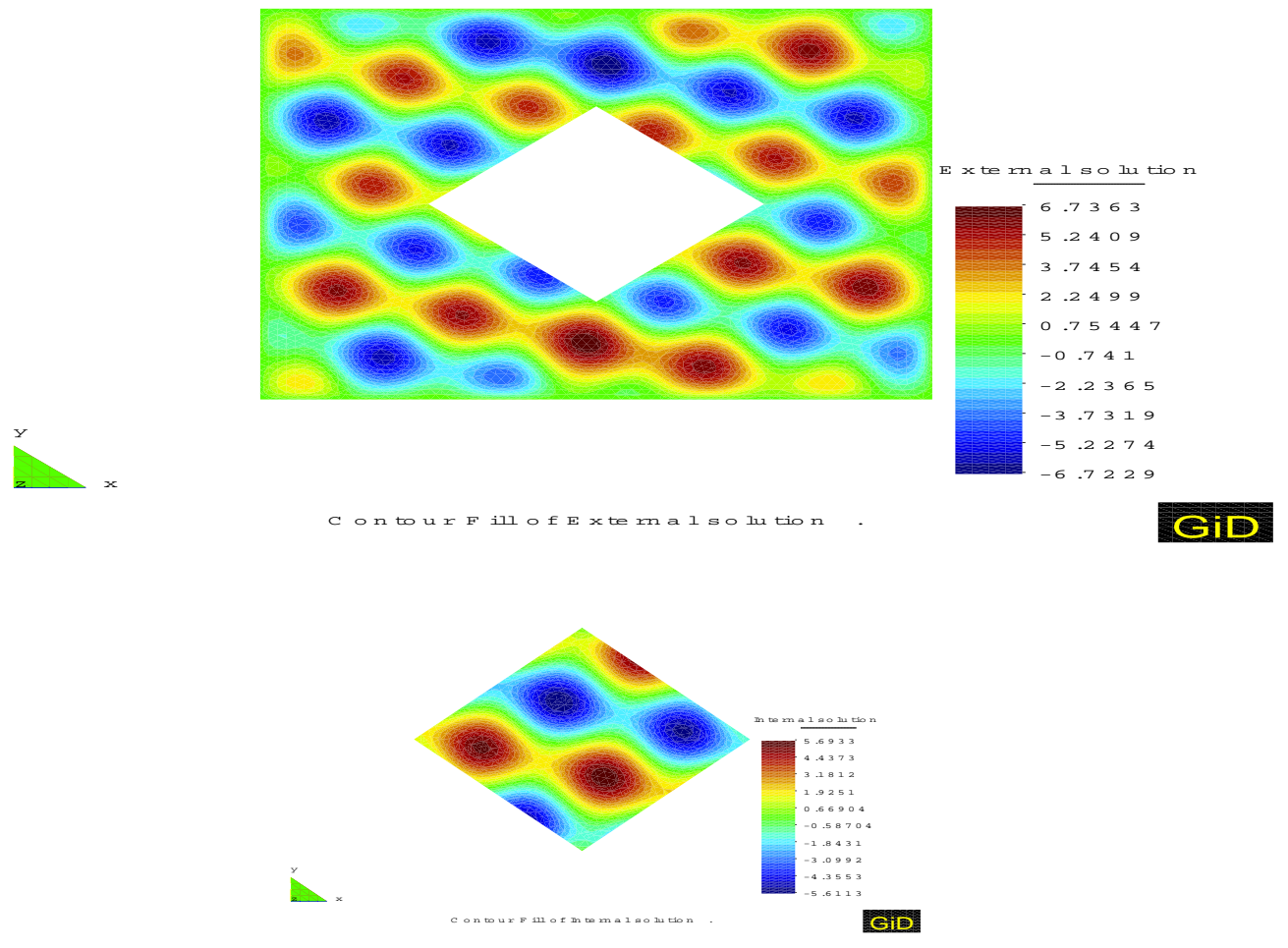

Figure 15: Square box with a floating subdomain: computed solution on $\Omega_{i}$ and on $\Omega_{e}$ for the finest mesh of 687 and 4621 nodes respectively. 


\section{References}

[1] R.A. Adams, Sobolev Spaces. Pure and Applied Mathematics, Vol. 65. Academic Press, New York-London, 1975.

[2] C. Bacuta, A unified approach for Uzawa algorithm. SIAM J. Numer. Anal., 44 (2006), 2633-2649.

[3] H.J.C. Barbosa, T.J.R. Hughes, Boundary Lagrange multipliers in finite element methods: error analysis in natural norms. Numerische Mathematik, 62 (1992), 1-15.

[4] F. Ben Belgacem, The Mortar finite element method with Lagrange multipliers. Numer. Math., 84 (1999), 173-197.

[5] S. Bertoluzza, Wavelet stabilization: the Lagrange multiplier method. Numer. Math., 86 (2000), 1-28.

[6] C. Bernardi, Y. Maday, A.T. Patera, A new nonconforming approach to domain decomposition : the mortar element method. Collège de France Seminar XI, H. Brezis \& J.-L. Lions eds., Pitman (1994), 13-51.

[7] C. Bernardi, Y. Maday, F. Rapetti, Discrétisations variationnelles de problèmes aux limites elliptiques. Mathématiques \& Applications, 45. Springer-Verlag, New-York, 2002.

[8] M. Bhardwaj, D. Day, C. Farhat, M. Lesoinne, K. Pierson, D. Rixen, Application of the FETI method to ASCI problems, scalability results on 1000 processors and discussion of highly heterogeneous problems. Internat. J. Numer. Methods Engrg., 47 (2000), 513-535.

[9] M.R. Dorr, Domain decomposition via Lagrange multipliers. UCRL98532, Lawrence Livermore National laboratory, Livermore, 1988.

[10] C. Farhat, A Lagrange multiplier based divide and conquer finite element algorithm. J. Comput. Systems Engrg., 2 (1991), 149-156.

[11] C. Farhat, J. Mandel, F.-X. Roux, Optimal convergence properties of the FETI domain decomposition method. Comput. Methods Appl. Mech. Engrg., 115 (1994), 364-385 . 
[12] C. Farhat, F.-X. Roux, A method of finite element tearing and interconnecting and its parallel solution algorithm. Int. J. Numer. Methods Engrg., 32 (1991), 1205-1227.

[13] C. Farhat, F.-X. Roux, An unconventional domain decomposition method for an efficient parallel solution of large-scale finite element systems. SIAM J. Sci. Statist. Comput., 13 (1992), 379-396.

[14] C. Farhat, M. Lesoinne, P. LeTallec, K. Pierson, D. Rixen, FETI-DP: a dualprimal unified FETI method, Part I: A faster alternative to the two-level FETI method. Int. J. Numer. Methods Engrg., 50 (2001), 15231544 .

[15] V. Girault, P.-A. Raviart, Finite Element Methods for Navier-Stokes Equations. Theory and Algorithms. Springer Series in Computational Mathematics, 5. Springer-Verlag, Berlin, 1986.

[16] R. Glowinski, Q.V. Dinh, J. Periaux, Domain decomposition methods for nonlinear problems in fluid dynamics. Comput. Methods Appl. Mech. Engrg., 40 (1983), 27-109.

[17] P. Grisvard, Singularities in Boundary value problems. Recherches en Mathématiques Appliquées, 22. Masson, 1992.

[18] J. Mandel, R. Tezaur, On the convergence of a dual-primal substructuring method. Numer. Math., 88 (2001), 543-558.

[19] J. Mandel, R. Tezaur, Convergence of a substructuring method with Lagrange multipliers. Numer. Math., 73 (1996), 473-487.

[20] J. Mandel, R. Tezaur, C. Farhat, A scalable substructuring method by Lagrange multipliers for plate bending problems. SIAM J. Numer. Anal., 36 (1999), 1370-1391.

[21] A. Quarteroni, R. Sacco, F. Saleri, Numerical Mathematics. Texts in Applied Mathematics, vol. 37, Springer-Verlag, New-York, 2nd edition, 2006.

[22] P.-A. Raviart, J.-M. Thomas, Primal Hybrid Finite Element Methods for second order eliptic equations. Math. Comp., 31 (1977), 391-413. 
[23] D. J. Rixen, C. Farhat, R. Tezaur, J. Mandel, Theoretical comparison of the FETI and algebraically partitioned FETI methods, and performance comparisons with a direct sparse solver. Int. J. Numer. Methods Engrg., 46 (1999), 501-533.

[24] F.-X. Roux, Méthode de décomposition de domaine à l'aide de multiplicateurs de Lagrange et application à la résolution en parallèle des équations de l'élasticité linéaire. Thesis, Université Pierre et Marie Curie (1989).

[25] F.-X. Roux, Acceleration of the outer conjugate gradient by reorthogonalization for a domain decomposition method for structural analysis problems. C.R. Acad. Sci. Paris Sér. I Math., 308 (1989), 193-198. 